TRANSACTIONS OF THE

AMERICAN MATHEMATICAL SOCIETY

Volume 358, Number 6, Pages 2713-2734

S 0002-9947(05)03826-2

Article electronically published on December 20, 2005

\title{
THETA LIFTING OF NILPOTENT ORBITS FOR SYMMETRIC PAIRS
}

\author{
KYO NISHIYAMA, HIROYUKI OCHIAI, AND CHEN-BO ZHU
}

Dedicated to Roger Howe on his sixtieth birthday

\begin{abstract}
We consider a reductive dual pair $\left(G, G^{\prime}\right)$ in the stable range with $G^{\prime}$ the smaller member and of Hermitian symmetric type. We study the theta lifting of nilpotent $K_{\mathbb{C}^{-}}^{\prime}$-orbits, where $K^{\prime}$ is a maximal compact subgroup of $G^{\prime}$ and we describe the precise $K_{\mathbb{C}}$-module structure of the regular function ring of the closure of the lifted nilpotent orbit of the symmetric pair $(G, K)$. As an application, we prove sphericality and normality of the closure of certain nilpotent $K_{\mathbb{C}}$-orbits obtained in this way. We also give integral formulas for their degrees.
\end{abstract}

\section{INTRODUCTION}

Let $\left(G, G^{\prime}\right)$ be a reductive dual pair in a symplectic group $\mathbb{G}=S p(2 N, \mathbb{R})$, where $N$ denotes the rank of $\mathbb{G}$. Throughout this paper, we will assume that $\left(G, G^{\prime}\right)$ is of type I, and it is in the stable range with $G^{\prime}$ the smaller member (cf. 9, 18). We will also assume that $G^{\prime} / K^{\prime}$ is an irreducible Hermitian symmetric space. By the classification of irreducible dual pairs, our restriction amounts to saying that $\left(G, G^{\prime}\right)$ is in the following list (cf. [11).

TABLE 1. The dual pairs treated in this paper

\begin{tabular}{ll} 
the pair $\left(G, G^{\prime}\right)$ & stable range condition \\
\hline$(O(p, q), S p(2 n, \mathbb{R}))$ & $2 n<\min (p, q)$ \\
$(U(p, q), U(m, n))$ & $m+n \leq \min (p, q)$ \\
$\left(S p(p, q), O^{*}(2 n)\right)$ & $n \leq \min (p, q)$
\end{tabular}

Note that we have excluded the equality $2 n=\min (p, q)$ in the first case, to avoid some small technicalities.

Let $\mathfrak{g}\left(\right.$ resp. $\left.\mathfrak{g}^{\prime}\right)$ be the complexification of the Lie algebra of $G$ (resp. $\left.G^{\prime}\right)$ and let $K$ (resp. $K^{\prime}$ ) be a maximal compact subgroup of $G$ (resp. $\left.G^{\prime}\right)$. Let

$$
\mathfrak{g}=\mathfrak{k} \oplus \mathfrak{s}, \quad \mathfrak{g}^{\prime}=\mathfrak{k}^{\prime} \oplus \mathfrak{s}^{\prime},
$$

Received by the editors December 18, 2003, and, in revised form, August 14, 2004

2000 Mathematics Subject Classification. Primary 22E46, 11 F27.

Key words and phrases. Reductive dual pair, theta lifting, nilpotent orbits, harmonic polynomial, invariant theory. 
be respectively Cartan decompositions of $\mathfrak{g}$ and $\mathfrak{g}^{\prime}$, compatible in a certain way (see \$2).

Notation. Let $G$ be a reductive algebraic group acting on an affine variety $X$. Then we denote the ring of $G$-invariants on the coordinate ring by $\mathbb{C}[X]^{G}$, and the affine quotient of $X$ by $G$ is defined to be $X / / G=\operatorname{Spec} \mathbb{C}[X]^{G}$, which parameterizes closed orbits in $X$. The affine variety $X / / G$ is often called a categorical quotient.

Let $W_{\mathbb{R}} \simeq \mathbb{R}^{2 N}$ be a real symplectic space which realizes $\mathbb{G}=S p(2 N, \mathbb{R})$ as a symplectic group on $W_{\mathbb{R}}$. There is a canonical complex structure on $W_{\mathbb{R}}$ and we can view $W_{\mathbb{R}}$ as (the underlying real vector space of) a complex vector space $W \simeq \mathbb{C}^{N}$. The symplectic form on $W_{\mathbb{R}}$ is then given by the imaginary part of a canonical positive definite Hermitian form on $W$. By this identification, a maximal compact subgroup $\mathbb{K}$ of $\mathbb{G}$ is realized as the unitary group $U(W) \simeq U(N)$ on $W \simeq \mathbb{C}^{N}$.

We may choose maximal compact subgroups $K$ and $K^{\prime}$ of $G$ and $G^{\prime}$ respectively, in such a way that $K \cdot K^{\prime}$ is contained in the standard maximal compact subgroup $\mathbb{K} \simeq U(N)$ of $\mathbb{G}$.

There is a double fibration of $W$ by the moment maps $\varphi$ and $\psi$ :

$$
\mathfrak{s} \stackrel{\varphi}{\longleftarrow} W \stackrel{\psi}{\longrightarrow} \mathfrak{s}^{\prime} .
$$

For explicit realization of these maps, see the Appendix. Here we only mention some of the important properties of $\varphi$ and $\psi$. Thanks to the stable range condition, $\psi$ is a surjective, affine quotient map by the action of $K_{\mathbb{C}}$. Thus we have $\mathfrak{s}^{\prime} \simeq W / / K_{\mathbb{C}}$. Furthermore, $\psi: W \rightarrow \mathfrak{s}^{\prime}$ is flat. On the other hand, the image of $\varphi$ is an irreducible closed subvariety of $\mathfrak{s}$ and $\varphi: W \rightarrow \varphi(W) \subset \mathfrak{s}$ is also an affine quotient map by $K_{\mathbb{C}}^{\prime}$.

Theorem A (Theorem [2.5). Take a nilpotent $K_{\mathbb{C}}^{\prime}$-orbit $\mathbb{O}^{\prime} \subset \mathcal{N}\left(\mathfrak{s}^{\prime}\right)$. Then the scheme theoretic fiber product $\psi^{-1}\left(\overline{\mathbb{O}^{\prime}}\right)=W \times_{\mathfrak{s}^{\prime}} \overline{\mathbb{O}^{\prime}}$ is a reduced, closed irreducible affine subvariety of $W$. Moreover, it is the closure of a single $K_{\mathbb{C}} \times K_{\mathbb{C}}^{\prime}$-orbit in $W$.

The above result easily implies that the set $\varphi\left(\psi^{-1}\left(\overline{\mathbb{O}^{\prime}}\right)\right)$ is the closure of a single

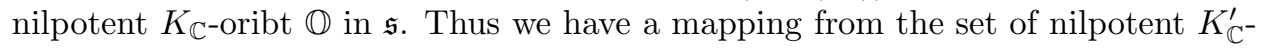

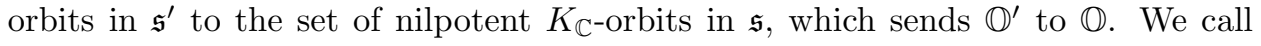
this mapping the theta lifting and denote it by $\mathbb{O}=\theta\left(\mathbb{O}^{\prime}\right)$. In the language of the signed Young diagrams, $\mathbb{O}$ is obtained from $\mathbb{O}^{\prime}$ by adding one extra box to each row at the right end (see Proposition 2.12). Thus $\mathbb{O}$ is slightly "bigger" than $\mathbb{O}^{\prime}$.

We remark that T. Ohta has proved the irreducibility and the existence of an open dense orbit by a totally different (but case-by-case) method (24], 25]). Another recent work by Daszkiewicz, Kraśkiewicz and Przebinda 6] proves similar results by classifying $K_{\mathbb{C}} \times K_{\mathbb{C}}^{\prime}$-orbit in $W$ combinatorially. There have also been earlier investigations of correspondence of complex nilpotent orbits in [5] and by S.-Y. Pan for complex as well as real nilpotent orbits.

Our approach is in some sense intrinsic and it is based on methods of algebraic geometry. More importantly, we hope that it will help to shed light on the geometric aspects of the theta correspondence. The recent work of two of the authors [22, 23] are efforts in this direction.

Let $\mathbb{C}[\overline{\mathbb{O}}]$ (resp. $\left.\mathbb{C}\left[\overline{\mathbb{O}^{\prime}}\right]\right)$ be the regular function ring on the closure of $\mathbb{O}\left(\right.$ resp. $\left.\mathbb{O}^{\prime}\right)$.

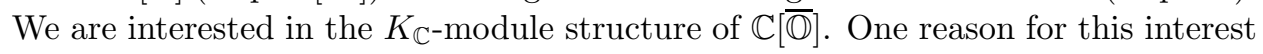
comes from Vogan's philosophy of unipotent representations [30. Very roughly speaking, it entails that if a unitary representation $\pi$ is to be "associated" to a 
nilpotent orbit $\mathbb{O}$, the regular function ring $\mathbb{C}[\overline{\mathbb{O}}]$ should encode the $K$-structure of the representation $\pi$. In the present situation, we will show that the $K_{\mathbb{C} \text {-module }}$ structure of $\mathbb{C}[\overline{\mathbb{O}}]$ can be described entirely and explicitly by the regular function

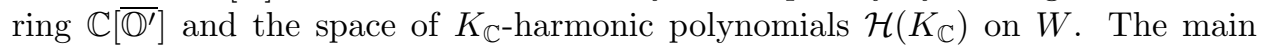
results are summarized in Theorem 2.10. In particular, we have the following.

Theorem B. We have a $K_{\mathbb{C} \text {-module isomorphism }}$

$$
\mathbb{C}[\overline{\mathbb{O}}] \simeq\left(\mathcal{H}\left(K_{\mathbb{C}}\right) \otimes \mathbb{C}\left[\overline{\mathbb{O}^{\prime}}\right]\right)^{K_{\mathbb{C}}^{\prime}}
$$

It is well known 10 that $\mathcal{H}\left(K_{\mathbb{C}}\right)$ is multiplicity-free as a representation of $K_{\mathbb{C}} \times L_{\mathbb{C}}^{\prime}$ and the decomposition of $\left.\mathcal{H}\left(K_{\mathbb{C}}\right)\right|_{K_{\mathbb{C}} \times L_{\mathbb{C}}^{\prime}}$ determines a one-to-one correspondence between irreducible representations of $K_{\mathbb{C}}$ and $L_{\mathbb{C}}^{\prime}$ occurring in $\mathcal{H}\left(K_{\mathbb{C}}\right)$. Here $L^{\prime}=$ $K^{\prime} \times K^{\prime}$. We note that the form of isomorphism $\mathbb{C}[\overline{\mathbb{O}}] \simeq\left(\mathcal{H}\left(K_{\mathbb{C}}\right) \otimes \mathbb{C}\left[\overline{\mathbb{O}^{\prime}}\right]\right)^{K_{\mathbb{C}}^{\prime}}$ bears strong resemblance to theta correspondence of admissible representations of $G^{\prime}$ and $G$ [1].

In 43, we will give some examples of the lifted orbits and their regular function rings. We obtain a family of spherical nilpotent orbits and a precise $K_{\mathbb{C} \text {-module }}$ structure of $\mathbb{C}[\overline{\mathbb{O}}]$. We note that they occupy a large portion of the set of all the

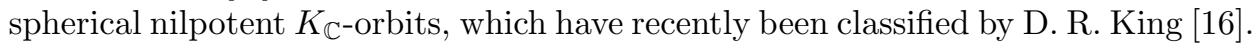
As an application we prove normality of the closure of some of these nilpotent orbits, which are lifted from the smaller group. We also give explicit integral formulas of their degrees. The result is summarized in Proposition 3.5. The formulas involve a certain integral expression, which can be explicitly evaluated.

Theorem C. Let $\kappa$ be a complex number with a positive real part. Then

$$
\frac{1}{n !} \int_{\Omega_{n}} D_{n}\left(x^{2}\right) D_{n}(x) \prod_{i=1}^{n} x_{i}^{\kappa-1} d x=\frac{2^{n(n-1) / 2} \prod_{i=0}^{n-1} i ! \Gamma(\kappa+2 i)}{\Gamma(3 n(n-1) / 2+n \kappa+1)}
$$

where $D_{n}(x)=\prod_{1 \leq i<j \leq n}\left(x_{i}-x_{j}\right), D_{n}\left(x^{2}\right)=\prod_{1 \leq i<j \leq n}\left(x_{i}^{2}-x_{j}^{2}\right)$, and the region of integral is given as $\Omega_{n}=\left\{x=\left(x_{i}\right)_{1 \leq i \leq n} \mid x_{i} \geq 0, \sum_{i=1}^{\bar{n}} x_{i} \leq 1\right\}$.

In the Appendix, we describe the explicit form of moment maps and give some basic properties of null cones. The results in the Appendix are known to the experts, but we include them because of lack of appropriate references.

\section{DiAMOND PAIRS}

We review certain structure results related to our dual pairs [1].

Let $W_{\mathbb{R}} \simeq \mathbb{R}^{2 N}$ be a real symplectic space which realizes $\mathbb{G}=S p(2 N, \mathbb{R})$ as a symplectic group on $W_{\mathbb{R}}$. There is a canonical complex structure on $W_{\mathbb{R}}$ and we can view $W_{\mathbb{R}}$ as (the underlying real vector space of) a complex vector space $W \simeq \mathbb{C}^{N}$. The symplectic form on $W_{\mathbb{R}}$ is then given by the imaginary part of a canonical positive definite Hermitian form on $W$. By this identification, a maximal compact subgroup $\mathbb{K}$ of $\mathbb{G}$ is realized as the unitary group $U(W) \simeq U(N)$ on $W \simeq \mathbb{C}^{N}$.

We may choose maximal compact subgroups $K$ and $K^{\prime}$ of $G$ and $G^{\prime}$ respectively, in such a way that $K \cdot K^{\prime}$ is contained in the standard maximal compact subgroup $\mathbb{K} \simeq U(N)$ of $\mathbb{G}$.

In view of Table1, we sometimes write $G=G(p, q)$ for $O(p, q), U(p, q)$ or $S p(p, q)$. We denote simply $G(k)=G(k, 0)$, which is compact.

From the pair $\left(G, G^{\prime}\right)$, we define another three dual pairs, which form so-called diamond dual pairs (see [11, §5]). Namely, take the commutant of $K$ in $\mathbb{G}$ and 
denote it by $M^{\prime}$. Then $M^{\prime}$ is also of Hermitian symmetric type and isomorphic to $G^{\prime} \times G^{\prime}$ containing $G^{\prime}$ as the diagonal. The pair $\left(K, M^{\prime}\right)$ is a dual pair of compact type. Also, take the full commutant of $K$ in $\mathbb{K}$, and denote it by $L^{\prime}$. Then $L^{\prime}$ is a maximal compact subgroup of $M^{\prime}$, and it is isomorphic to $K^{\prime} \times K^{\prime}$ and contains $K^{\prime}$ as a diagonal subgroup. Similarly, define $M$ as the commutant of $K^{\prime}$ in $\mathbb{G}$, and $L$ the commutant in $\mathbb{K}$. Let us summarize this somewhat complicated situation in Figure 1.

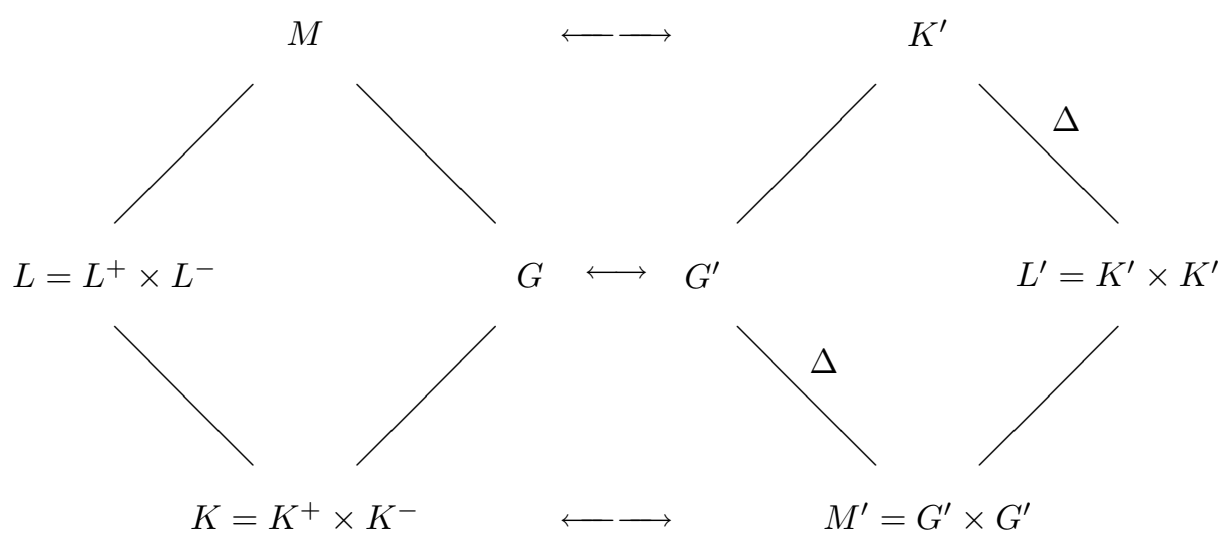

Figure 1. The diagram of a diamond pair

An explicit description of the diamond pairs for our three cases is given in [11, (5.3)]. In the table there, $L$ (resp. $L^{\prime}$ ) in our notation is written as $M^{(1,1)}$ (resp. $\left.M^{\prime(1,1)}\right)$. For convenience of readers, we reproduce the table here (Table 2) with some additional remarks.

TABLE 2. Three diamond dual pairs

\begin{tabular}{ccccc}
$\left(G, G^{\prime}\right)$ & $M$ & $K=K^{+} \times K^{-}$ & $L=L^{+} \times L^{-}$ & $K^{\prime}$ \\
\hline$(O(p, q), S p(2 n, \mathbb{R}))$ & $U(p, q)$ & $O(p) \times O(q)$ & $U(p) \times U(q)$ & $U(n)$ \\
$(U(p, q), U(m, n)))$ & $U(p, q) \times U(p, q)$ & $U(p) \times U(q)$ & $(U(p) \times U(p)) \times(U(q) \times U(q))$ & $U(m) \times U(n)$ \\
$\left(S p(p, q), O^{*}(2 n)\right)$ & $U(2 p, 2 q)$ & $S p(p) \times S p(q)$ & $U(2 p) \times U(2 q)$ & $U(n)$ \\
& $M^{\prime}=G^{\prime} \times G^{\prime}$, & $L^{\prime}=K^{\prime} \times K^{\prime}$ &
\end{tabular}

Note that $M / L$ is a Hermitian symmetric space (not necessarily irreducible), and the containment $M \supset G$ (resp. $L \supset K$ ) may or may not be the diagonal map, but it is always a symmetric pair.

Recall $\mathbb{G}=S p\left(W_{\mathbb{R}}\right)$ and the complex vector space $W \simeq \mathbb{C}^{N}$ which is identical to $W_{\mathbb{R}}$ as a real vector space. Then there exists a direct sum decomposition $W_{\mathbb{R}}=$ $W_{\mathbb{R}}^{+} \oplus W_{\mathbb{R}}^{-}$and correspondingly $W=W^{+} \oplus W^{-}$, which are compatible with direct product decompositions $L=L^{+} \times L^{-}$and $K=K^{+} \times K^{-}$in the following way. The subgroups $L^{ \pm}$and $K^{ \pm}$are contained in the unitary group $U\left(W^{ \pm}\right)$. Moreover, the 
pairs $\left(K^{ \pm}, G^{\prime}\right)$ and $\left(L^{ \pm}, K^{\prime}\right)$ are dual pairs in $S p\left(W_{\mathbb{R}}^{ \pm}\right)$. Also note that $L^{ \pm} \supset K^{ \pm}$ is a symmetric pair.

For explicit realization of these decompositions, see the Appendix.

\section{Theta lift of A nilpotent orbit}

Let $\mathfrak{G}=\operatorname{Lie}(\mathbb{G})_{\mathbb{C}}\left(\right.$ resp. $\left.\mathfrak{K}=\operatorname{Lie}(\mathbb{K})_{\mathbb{C}}\right)$ be the complexified Lie algebra of $\mathbb{G}=$ $S p(2 N, \mathbb{R})$ (resp. $\mathbb{K}=U(N))$. Let $\mathfrak{G}=\mathfrak{K} \oplus \mathfrak{P}$ be the corresponding (complexified) Cartan decomposition. Note that the complexified Lie group $\mathbb{K}_{\mathbb{C}}$ acts on the Cartan space $\mathfrak{P}$ by the restriction of the adjoint action; under this action $\mathfrak{P}$ breaks up into $\mathfrak{P}_{ \pm}$, each of which can be identified with a copy of the space $\operatorname{Sym}_{N}(\mathbb{C})$ of complex symmetric matrices of size $N$. The action of $k \in G L(N, \mathbb{C}) \simeq \mathbb{K}_{\mathbb{C}}$ on $\mathfrak{P}=\mathfrak{P}_{+} \oplus \mathfrak{P}_{-} \simeq \operatorname{Sym}_{N}(\mathbb{C}) \oplus \operatorname{Sym}_{N}(\mathbb{C})$ is given by

$$
k \cdot(X, Y)=\left(k X^{t} k,{ }^{t} k^{-1} Y k^{-1}\right) \quad\left(X, Y \in \operatorname{Sym}_{N}(\mathbb{C})\right) .
$$

Let $\mathbb{O}_{\text {min }} \subseteq \mathfrak{P}_{+}$be the minimal nilpotent $\mathbb{K}_{\mathbb{C}} \simeq G L(N, \mathbb{C})$ orbit in $\mathfrak{P}_{+}$. We have the identification $\mathbb{O}_{\text {min }}=\left\{(X, 0) \mid X \in \operatorname{Sym}_{N}(\mathbb{C}), \operatorname{rank} X=1\right\}$. It is also well known that there is a map

$$
\begin{aligned}
& j: \quad W \quad \longrightarrow \quad \overline{\mathbb{O}_{\text {min }}} \\
& \stackrel{\Psi}{w} \longmapsto\left({ }^{t} w w, 0\right)
\end{aligned}
$$$$
w=\left(w_{1}, \ldots, w_{N}\right) \in \mathbb{C}^{N}=W
$$

by which $\overline{\mathbb{O}_{\text {min }}}=\mathbb{O}_{\text {min }} \cup\{0\}$ is regarded as a geometric quotient of the space $W$ by

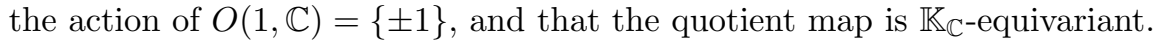

Let us consider the dual pair $\left(G, G^{\prime}\right) \subseteq \mathbb{G}$. The maximal compact subgroup $K$ (resp. $K^{\prime}$ ) determines a Cartan decomposition of the complexified Lie algebra $\mathfrak{g}$ (resp. $\left.\mathfrak{g}^{\prime}\right)$ of $G\left(\right.$ resp. $\left.G^{\prime}\right)$ :

$$
\mathfrak{g}=\mathfrak{k} \oplus \mathfrak{s}, \quad \mathfrak{g}^{\prime}=\mathfrak{k}^{\prime} \oplus \mathfrak{s}^{\prime},
$$

where $\mathfrak{k}$ (resp. $\left.\mathfrak{k}^{\prime}\right)$ is the complexified Lie algebra of $K$ (resp. $K^{\prime}$ ), and $\mathfrak{s}\left(\right.$ resp. $\left.\mathfrak{s}^{\prime}\right)$ is the orthogonal complement of $\mathfrak{k}$ (resp. $\mathfrak{k}^{\prime}$ ) with respect to the Killing form. The subspace $\mathfrak{s}\left(\right.$ resp. $\left.\mathfrak{s}^{\prime}\right)$ carries a linear action of $K_{\mathbb{C}}\left(\right.$ resp. $\left.K_{\mathbb{C}}^{\prime}\right)$ via the restriction of the adjoint representation. Since $G^{\prime} / K^{\prime}$ is a Hermitian symmetric space by assumption, $\mathfrak{s}^{\prime}$ breaks up into irreducible pieces $\mathfrak{s}^{\prime}{ }_{ \pm}$under the action of $K_{\mathbb{C}}^{\prime}$.

We can arrange the Cartan decompositions so that $\mathfrak{s} \oplus \mathfrak{s}^{\prime}$ is contained in $\mathfrak{P}$. Then the inclusion map $\mathfrak{s} \subset \mathfrak{P}\left(\right.$ resp. $\left.\mathfrak{s}^{\prime} \subset \mathfrak{P}\right)$ induces the projection $\mathfrak{P}^{*} \rightarrow \mathfrak{s}^{*}$ $\left(\right.$ resp. $\left.\mathfrak{P}^{*} \rightarrow \mathfrak{s}^{\prime *}\right)$. By identifying the complex linear dual $\mathfrak{P}^{*}\left(\right.$ resp. $\left.\mathfrak{s}^{*}, \mathfrak{s}^{\prime *}\right)$ with $\mathfrak{P}$ (resp. $\left.\mathfrak{s}, \mathfrak{s}^{\prime}\right)$ by the invariant trace form of matrices, we obtain two projection maps $P_{\mathfrak{s}}: \mathfrak{P} \rightarrow \mathfrak{s}$ and $P_{\mathfrak{s}^{\prime}}: \mathfrak{P} \rightarrow \mathfrak{s}^{\prime}$. The projections restricted to $\overline{\mathbb{O}_{\text {min }}}$ will then induce the so-called moment maps (cf. [24]):

$$
\begin{aligned}
& \varphi: W \stackrel{\begin{array}{c}
\text { geom. quotient } \\
\text { by }\{ \pm 1\}
\end{array}}{\longrightarrow} \overline{\mathbb{O}_{\text {min }}} \stackrel{\text { projection }}{\longrightarrow} \mathfrak{s}, \\
& \psi: W \longrightarrow \mathfrak{\mathbb { O }}_{\text {min }} \longrightarrow
\end{aligned}
$$

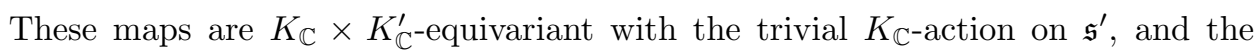
trivial $K_{\mathbb{C}}^{\prime}$-action on $\mathfrak{s}$ respectively. Also we can (and we will) choose the subspaces $\mathfrak{s}^{\prime}{ }_{ \pm} \subset \mathfrak{s}^{\prime}$ and $W^{ \pm} \subset W$ so that $\psi\left(W^{ \pm}\right) \subset \mathfrak{s}^{\prime}{ }_{ \pm}$holds (cf. $\llbracket$ for the notation $W^{ \pm}$).

For a set $S \subseteq \mathfrak{P}$, we denote by $\mathcal{N}(S)$ the subset of all nilpotent elements in $S$. 
Lemma 2.1. We can choose Cartan decompositions and moment maps so that the following conditions hold:

(1) $\psi$ is an affine quotient map by $K_{\mathbb{C}}$ onto $\mathfrak{s}^{\prime}$, i.e., $\mathfrak{s}^{\prime} \simeq W / / K_{\mathbb{C}}$.

(2) $\varphi$ is an affine quotient map by $K_{\mathbb{C}}^{\prime}$ onto its closed image.

(3) $\varphi\left(\psi^{-1}\left(\mathcal{N}\left(\mathfrak{s}^{\prime}\right)\right)\right) \subset \mathcal{N}(\mathfrak{s})$.

Remark 2.2. For part (1) to hold true, we only need to have the following conditions: $\min (p, q) \geq n$ for $(O(p, q), S p(2 n, \mathbb{R})), \min (p, q) \geq \min (m, n)$ for $(U(p, q), U(m, n))$, and $\min (p, q) \geq \frac{n}{2}$ for $\left(S p(p, q), O^{*}(2 n)\right)$. They are much weaker than the stable range condition.

Proof. To show that $\psi: W \rightarrow \mathfrak{s}^{\prime}$ is an affine quotient map, it suffices to show that $\psi$ induces an algebra isomorphism $\mathbb{C}\left[\mathfrak{s}^{\prime}\right] \simeq \mathbb{C}[W]^{K_{\mathbb{C}}}$, where $\mathbb{C}[W]^{K_{\mathbb{C}}}$ denotes the space of invariants of the polynomial ring $\mathbb{C}[W]$. Clearly, it suffices to show that the restrictions $\left.\psi\right|_{W^{ \pm}}$induce algebra isomorphisms $\mathbb{C}\left[\mathfrak{s}^{\prime}{ }_{ \pm}\right] \simeq \mathbb{C}\left[W^{ \pm}\right]^{K_{\mathbb{C}}^{ \pm}}$.

Similarly, statement (2) asserts that the induced algebra homomorphism $\varphi^{*}$ : $\mathbb{C}[\mathfrak{s}] \rightarrow \mathbb{C}[W]$ maps $\mathbb{C}[\mathfrak{s}]$ onto the $K_{\mathbb{C}}^{\prime}$-invariants $\mathbb{C}[W]^{K_{\mathbb{C}}^{\prime}}$. Note that the closed subvariety $\operatorname{Im} \varphi \subset \mathfrak{s}$ is then defined by the ideal of relations of generators of invariants, or in other words, the kernel of $\varphi^{*}$.

Explicit constructions of moment maps for each case of the three dual pairs in Table 1 are summarized in the Appendix. From the explicit formulas given there, the statements (1)-(3) follow easily from classical invariant theory [31.

Using the moment maps, we shall define the theta lift of nilpotent orbits from $\mathcal{N}\left(\mathfrak{s}^{\prime}\right)$ to $\mathcal{N}(\mathfrak{s})$. Before that, we review the following well-known result due to Kostant.

We consider the null cones

$$
\mathfrak{N}=\psi^{-1}(0) \subset W, \quad \mathfrak{N}^{ \pm}=\left(\psi^{ \pm}\right)^{-1}(0) \subset W^{ \pm},
$$

where $\psi^{ \pm}: W^{ \pm} \rightarrow \mathfrak{s}_{ \pm}^{\prime}$ is the restriction of $\psi$ to $W^{ \pm}$. Let $J^{ \pm} \subset \mathbb{C}\left[W^{ \pm}\right]$be the augmentation ideal generated by homogeneous invariants $\mathbb{C}\left[W^{ \pm}\right]_{+}^{K_{\mathbb{C}}^{ \pm}}$of positive degree, and let $J=\mathbb{C}[W]_{+}^{K_{\mathbb{C}}} \cdot \mathbb{C}[W]=J^{+} \otimes J^{-}$.

We observe that the stable range condition implies that

(1) $J^{ \pm}$is the ideal in $\mathbb{C}\left[W^{ \pm}\right]$vanishing on $\mathfrak{N}^{ \pm}$and $J^{ \pm}$is prime;

(2) The single $K_{\mathbb{C}}^{ \pm}$-orbit of generic elements of $\mathfrak{N}^{ \pm}$is dense in $\mathfrak{N}^{ \pm}$.

See for example Proposition 3.7.3.7 and Theorem 3.8.6.4 in [12, and references therein. Some relevant properties of the null cones may also be found in the Appendix.

As usual, we denote by $\mathcal{H}\left(K_{\mathbb{C}}^{ \pm}\right)$the space of harmonic polynomials on $W^{ \pm}$(under the action of $K_{\mathbb{C}}^{ \pm}$). Similar notations apply throughout this article. Applying Kostant's criterion [17, we get

Theorem 2.3. Let $\left(G, G^{\prime}\right)$ be in the stable range (see Table 1) with $G^{\prime}$ the smaller member, and consider the dual pairs $\left(K^{ \pm}, G^{\prime}\right)$ in $S p\left(W_{\mathbb{R}}^{ \pm}\right)$. Then with respect to the action of $K_{\mathbb{C}}^{ \pm}$, we have a tensor product decomposition of $\mathbb{C}\left[W^{ \pm}\right]$:

$$
\mathbb{C}\left[W^{ \pm}\right] \simeq \mathcal{H}\left(K_{\mathbb{C}}^{ \pm}\right) \otimes \mathbb{C}\left[W^{ \pm}\right]^{K_{\mathbb{C}}^{ \pm}}
$$


Corollary 2.4. We have

$$
\mathbb{C}[W] \simeq \mathcal{H}\left(K_{\mathbb{C}}\right) \otimes \mathbb{C}[W]^{K_{\mathbb{C}}} \simeq \mathcal{H}\left(K_{\mathbb{C}}\right) \otimes \mathbb{C}\left[\mathfrak{s}^{\prime}\right]
$$

Consequently, the moment map $\psi: W \rightarrow \mathfrak{s}^{\prime}$ is flat.

Proof. Note that the induced map $\psi^{*}$ of the moment map $\psi$ is just an inclusion: $\psi^{*}: \mathbb{C}\left[\mathfrak{s}^{\prime}\right] \simeq \mathbb{C}[W]^{K_{\mathbb{C}}} \hookrightarrow \mathbb{C}[W]$. Since $\mathbb{C}[W] \simeq \mathcal{H}\left(K_{\mathbb{C}}\right) \otimes \mathbb{C}\left[\mathfrak{s}^{\prime}\right]$, it is free over $\mathbb{C}\left[\mathfrak{s}^{\prime}\right]$. This implies that $\psi$ is flat.

We are now in a position to prove

Theorem 2.5. Take a nilpotent $K_{\mathbb{C}^{\prime}}^{\prime}$ orbit $\mathbb{O}^{\prime} \subset \mathcal{N}\left(\mathfrak{s}^{\prime}\right)$. Then the scheme theoretic fiber product $\psi^{-1}\left(\overline{\mathbb{O}^{\prime}}\right)=W \times_{\mathfrak{s}^{\prime}} \overline{\mathbb{O}^{\prime}}$ is a reduced, closed irreducible affine subvariety of $W$. Moreover, it is the closure of a single $K_{\mathbb{C}} \times K_{\mathbb{C}}^{\prime}$-orbit in $W$.

Proof. Let us prove that the fiber $W \times_{\mathfrak{s}^{\prime}} \overline{\mathbb{O}^{\prime}}$ is reduced. For this, it is enough to show that the fiber of each closed point in $\mathfrak{s}^{\prime}$ is reduced. Namely, we prove the following lemma.

Lemma 2.6. Let $x \in \mathfrak{s}^{\prime}$ be a closed point. Then the scheme theoretic fiber $\psi^{-1}(x)=$ $W \times_{\mathfrak{s}^{\prime}}\{x\}$ is a closed, reduced and irreducible affine subvariety of $W$. Moreover, it is the closure of a single $K_{\mathbb{C}}$-orbit.

Proof. The scheme theoretic fiber is defined by $W \times_{\mathfrak{s}^{\prime}}\{x\}=\operatorname{Spec}\left(\mathbb{C}[W] \otimes_{\mathbb{C}\left[\mathfrak{s}^{\prime}\right]} \mathbb{C}_{x}\right)$. Here $\mathbb{C}_{x}=\mathbb{C}\left[\mathfrak{s}^{\prime}\right] / \mathfrak{m}_{x} \simeq \mathbb{C}$, where $\mathfrak{m}_{x}$ denotes the maximal ideal corresponding to the closed point $x$. Put $\mathcal{A}_{x}=\mathbb{C}[W] \otimes_{\mathbb{C}\left[\mathfrak{s}^{\prime}\right]} \mathbb{C}_{x}$. We are to show that $\mathcal{A}_{x}$ is an integral domain. The proof is based on the general argument about deformations of homogeneous integral domains.

Let us recall the tensor product decomposition $\mathbb{C}[W]=\mathcal{H}\left(K_{\mathbb{C}}\right) \otimes \mathbb{C}\left[\mathfrak{s}^{\prime}\right]$. Thus, if we abbreviate $\mathcal{H}=\mathcal{H}\left(K_{\mathbb{C}}\right)$, we can identify

$$
\mathcal{A}_{x}=\mathbb{C}[W] \otimes_{\mathbb{C}\left[\mathfrak{s}^{\prime}\right]} \mathbb{C}_{x}=\mathcal{H} \otimes \mathbb{C}_{x} \simeq \mathcal{H} .
$$

However, note that $\mathcal{H}$ is not an algebra but it only enjoys a structure of $K_{\mathbb{C}} \times K_{\mathbb{C}^{-}}^{\prime}$ module. Since $\mathcal{H}$ is a graded subspace of $\mathbb{C}[W]$, it is naturally graded by the ordinary degree of polynomials. We denote the grading by $\mathcal{H} \simeq \bigoplus_{k \geq 0} \mathcal{H}_{k}$. Then, $\mathcal{A}_{x}$ becomes a filtered algebra by putting

$$
\left(\mathcal{A}_{x}\right)_{i}=\sum_{k \leq i} \mathcal{H}_{k} \otimes 1 \subset \mathcal{H} \otimes \mathbb{C}_{x}=\mathcal{A}_{x}
$$

Now assume that $\mathcal{A}_{x}$ is not an integral domain. Then there are non-zero elements $a, b \in \mathcal{A}_{x}$ such that $a \cdot b=0$. Let us express $a$ and $b$ as

$$
a=\sum_{i=0}^{d} a_{i} \otimes 1 \in \mathcal{H} \otimes \mathbb{C}_{x} ; \quad b=\sum_{j=0}^{d^{\prime}} b_{j} \otimes 1 \in \mathcal{H} \otimes \mathbb{C}_{x},
$$

with $a_{d} \neq 0$ and $b_{d^{\prime}} \neq 0$. Then $a b=0$ implies $\left(a_{d} b_{d^{\prime}}\right) \otimes 1 \in\left(\mathcal{A}_{x}\right)_{d+d^{\prime}-1}$, but this is impossible. To see it, observe that the null cone $\mathfrak{N}=\mathfrak{N}^{+} \times \mathfrak{N}^{-}$is defined by the augmentation ideal $J$ which is prime, and so $\mathfrak{N}$ is irreducible. Note that $\mathbb{C}[\mathfrak{N}]=\mathbb{C}[W] / J \simeq \mathcal{H}$. Since $a_{d} b_{d^{\prime}} \notin \mathcal{H}$ by the irreducibility of the null cone, we have

which is a contradiction.

$$
a_{d} \cdot b_{d^{\prime}} \otimes 1 \notin \sum_{k=0}^{d+d^{\prime}-1} \mathcal{H}_{k} \otimes \mathbb{C}_{x}=\left(\mathcal{A}_{x}\right)_{d+d^{\prime}-1},
$$


Next, we prove that the fiber $\psi^{-1}(x)$ contains an open dense $K_{\mathbb{C} \text {-orbit. Put }}$ $M=\psi^{-1}(x)$ and denote by $\widehat{M}$ the asymptotic cone of $M$ (see [26, $\S 5.2$ ] for the definition of asymptotic cone). Then, by the flatness of $\psi$, the asymptotic cone $\widehat{M}$ coincides with the null cone $\mathfrak{N}$. Let $\mathcal{O}$ be a generic $K_{\mathbb{C}}$-orbit in $M$. Consider the cone $\mathbb{C} M$ generated by $M$ in $W$, then it is clear that the dimension of a generic orbit in $\mathbb{C} M$ is equal to $\operatorname{dim} \mathcal{O}$, which in turn coincides with the dimension of the generic orbit in $\overline{\mathbb{C} M} \subset W$. Since $\mathfrak{N}=\widehat{M} \subset \overline{\mathbb{C} M}$, the dimension of a generic orbit in $\mathfrak{N}$ cannot exceed that of $\mathcal{O}$. Note that $\mathfrak{N}$ has an open dense orbit (see the Appendix for details). This means that $\operatorname{dim} \mathcal{O} \geq \operatorname{dim} \mathfrak{N}$. On the other hand, we have an equality $\operatorname{dim} M=\operatorname{dim} \widehat{M}=\operatorname{dim} \mathfrak{N}$ of dimensions, hence $\operatorname{dim} \mathcal{O} \geq \operatorname{dim} M$. Since $\mathcal{O} \subset M$, we conclude that $\operatorname{dim} \mathcal{O}=\operatorname{dim} M$, and that $\mathcal{O}$ is an open dense orbit in $M$, by the irreducibility of $M$ just proved above.

Let us return to the proof of Theorem 2.5.

By $K_{\mathbb{C}}^{\prime}$-equivariancy of $\psi$, we get $\psi^{-1}\left(\mathbb{O}^{\prime}\right)=K_{\mathbb{C}}^{\prime} \cdot \psi^{-1}(\{x\})$ for any $x \in \mathbb{O}^{\prime}$. Consider the multiplication map $K_{\mathbb{C}}^{\prime} \times \psi^{-1}(\{x\}) \rightarrow \psi^{-1}\left(\mathbb{O}^{\prime}\right)$. Since $K_{\mathbb{C}}^{\prime} \times \psi^{-1}(\{x\})$ is irreducible by the above lemma, $\psi^{-1}\left(\mathbb{O}^{\prime}\right)$ is also irreducible (as an image of an irreducible set). Therefore its closure $\overline{\psi^{-1}\left(\mathbb{O}^{\prime}\right)}$ is irreducible. Since the moment map $\psi$ is flat by Corollary [2.4, it is an open map (8, Ex. (III.9.1)]). Thus we conclude that $\psi^{-1}\left(\overline{\mathbb{O}^{\prime}}\right)=\overline{\psi^{-1}\left(\mathbb{O}^{\prime}\right)}$ is irreducible.

By Lemma 2.6, $\psi^{-1}(x)$ contains an open dense $K_{\mathbb{C}^{-} \text {-orbit }} \mathcal{O}_{x}$. Then the union of $K_{\mathbb{C}^{-}}^{\prime}$-translates $K_{\mathbb{C}}^{\prime} \cdot \mathcal{O}_{x}$ is dense in $\psi^{-1}\left(\mathbb{O}^{\prime}\right)$. Thus $\overline{K_{\mathbb{C}}^{\prime} \cdot \mathcal{O}_{x}}=\overline{\psi^{-1}\left(\mathbb{O}^{\prime}\right)}=\psi^{-1}\left(\overline{\mathbb{O}^{\prime}}\right)$, which means that $K_{\mathbb{C}}^{\prime} \cdot \mathcal{O}_{x}$ is open in $\psi^{-1}\left(\overline{\mathbb{O}^{\prime}}\right)$.

Remark 2.7. As mentioned in the Introduction, T. Ohta proved the irreducibility and the existence of an open dense orbit by a totally different (but case-by-case) method (24, 25]). In fact, his method is applicable beyond the stable range. However, outside the stable range, things get much more complicated and the same statement of the above theorem is no longer true.

From the above theorem, we see that $\varphi\left(\psi^{-1}\left(\overline{\mathbb{O}^{\prime}}\right)\right)$ is a $K_{\mathbb{C}^{-} \text {-stable irreducible closed }}$ set in $\mathfrak{s}$. Since $\varphi\left(\psi^{-1}\left(\overline{\mathbb{O}^{\prime}}\right)\right)$ is contained in the nilpotent variety $\mathcal{N}(\mathfrak{s})$, which has

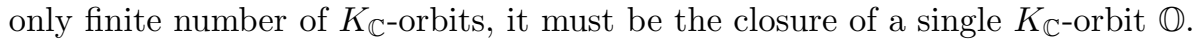

Definition 2.8. Let $\mathbb{O}^{\prime} \subset \mathfrak{s}^{\prime}$ be a nilpotent $K_{\mathbb{C}^{-}}^{\prime}$ orbit. Then $\psi^{-1}\left(\overline{\mathbb{O}^{\prime}}\right)$ is a closed, irreducible affine subvariety in $W$. The image $\varphi\left(\psi^{-1}\left(\overline{\mathbb{O}^{\prime}}\right)\right)$ is the closure of a single

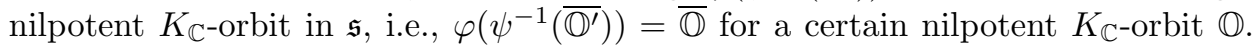
We call $\mathbb{O}$ the theta lift of $\mathbb{O}^{\prime}$, and denote it by $\theta\left(\mathbb{O}^{\prime}\right)$.

We introduce some notation. For any complex reductive Lie group $E$, let $\operatorname{Irr}(E)$ be the set of equivalence classes of irreducible finite dimensional representations of $E$. Given a (completely reducible) representation of $E$ on $\mathcal{U}$, let $\operatorname{Irr}(E ; \mathcal{U})$ be the subset of $\operatorname{Irr}(E)$ which appears in $\mathcal{U}$.

We now recall some standard results of Howe [10. As noted in the Introduction, the space of harmonics $\mathcal{H}\left(K_{\mathbb{C}}^{ \pm}\right)$is multiplicity-free as a representation of $K_{\mathbb{C}}^{ \pm} \times K_{\mathbb{C}}^{\prime}$. Furthermore, the decomposition

$$
\left.\mathcal{H}\left(K_{\mathbb{C}}^{+}\right)\right|_{K_{\mathbb{C}}^{+} \times K_{\mathbb{C}}^{\prime}} \simeq \sum_{\sigma \in \operatorname{Irr}\left(K_{\mathbb{C}}^{+} ; \mathcal{H}^{+}\right), \tau \in \operatorname{Irr}\left(K_{\mathbb{C}}^{\prime} ; \mathcal{H}^{+}\right)}^{\oplus} \sigma \otimes \tau
$$


determines a one-to-one correspondence between $\sigma \in \operatorname{Irr}\left(K_{\mathbb{C}}^{+} ; \mathcal{H}^{+}\right)$and $\tau \in$ $\operatorname{Irr}\left(K_{\mathbb{C}}^{\prime} ; \mathcal{H}^{+}\right)$, where we abbreviate $\mathcal{H}\left(K_{\mathbb{C}}^{+}\right)$to $\mathcal{H}^{+}$. Here (and in the sequel) $\sigma \otimes \tau$ denotes the tensor product of $\sigma$ and $\tau$, viewed as a representation of $K_{\mathbb{C}}^{+} \times K_{\mathbb{C}}^{\prime}$ (the external tensor product). Similar statements apply to $\mathcal{H}\left(K_{\mathbb{C}}^{-}\right)$.

We shall abbreviate $R^{ \pm}\left(K_{\mathbb{C}}^{\prime}\right)=\operatorname{Irr}\left(K_{\mathbb{C}}^{\prime} ; \mathcal{H}\left(K_{\mathbb{C}}^{ \pm}\right)\right)$in the following. Under the assumption of stable range, one can explicitly check that $\tau^{*} \in R^{+}\left(K_{\mathbb{C}}^{\prime}\right)$ if and only if $\tau \in R^{-}\left(K_{\mathbb{C}}^{\prime}\right)$, where $\tau^{*}$ denotes the contragredient representation of $\tau \in \operatorname{Irr}\left(K^{\prime}\right)$. So we put

$$
R\left(K_{\mathbb{C}}^{\prime}\right)=R^{+}\left(K_{\mathbb{C}}^{\prime}\right)^{*}=R^{-}\left(K_{\mathbb{C}}^{\prime}\right) .
$$

To summarize, for each $\tau \in R^{ \pm}\left(K_{\mathbb{C}}^{\prime}\right)$, there exists a unique $\sigma \in \operatorname{Irr}\left(K_{\mathbb{C}}^{ \pm}\right)$such that

$$
\operatorname{Hom}_{K_{\mathbb{C}}^{ \pm} \times K_{\mathbb{C}}^{\prime}}\left(\sigma \otimes \tau, \mathcal{H}\left(K_{\mathbb{C}}^{ \pm}\right)\right) \neq 0
$$

We denote this $\sigma$ by $\sigma^{ \pm}(\tau)$, specifying the dependency of $\tau$ and the sign \pm . Then, we can rewrite (2.2) and its sister statement as

$$
\mathcal{H}\left(K_{\mathbb{C}}^{+}\right) \simeq \sum_{\tau \in R\left(K_{\mathbb{C}}^{\prime}\right)}^{\oplus} \sigma^{+}\left(\tau^{*}\right) \otimes \tau^{*} \quad \text { and } \quad \mathcal{H}\left(K_{\mathbb{C}}^{-}\right) \simeq \sum_{\tau \in R\left(K_{\mathbb{C}}^{\prime}\right)}^{\oplus} \sigma^{-}(\tau) \otimes \tau .
$$

Remark 2.9. By the theory of highest weight, we may identify $R\left(K_{\mathbb{C}}^{\prime}\right)$ with a certain semigroup in the integral weight lattice of $K_{\mathbb{C}}^{\prime}$. We note that in an explicit realization, the semigroup $R\left(K_{\mathbb{C}}^{\prime}\right)$ may be identified with a set of partitions, and it is saturated. The explicit decompositions in (2.4) are well known. See [14 for the orthogonal and unitary cases, or [12] in general.

Theorem 2.10. Let $\left(G, G^{\prime}\right)$ be in the stable range (see Table1) with $G^{\prime}$ the smaller member. For a nilpotent $K_{\mathbb{C}}^{\prime}$-orbit $\mathbb{O}^{\prime} \subset \mathcal{N}\left(\mathfrak{s}^{\prime}\right)$, denote its theta lift by $\mathbb{O}=\theta\left(\mathbb{O}^{\prime}\right)$. Put $\Xi\left(\mathbb{O}^{\prime}\right)=\psi^{-1}\left(\overline{\mathbb{O}^{\prime}}\right)$. Then the closure $\overline{\mathbb{O}}$ is an affine quotient of $\Xi\left(\mathbb{O}^{\prime}\right)$ by $K_{\mathbb{C}}^{\prime}$.

$$
\overline{\mathbb{O}} \simeq \Xi\left(\mathbb{O}^{\prime}\right) / / K_{\mathbb{C}}^{\prime} .
$$

Moreover, the $K_{\mathbb{C}}$-module structure of the regular function ring of $\overline{\mathbb{O}}$ is given in terms of $\overline{\mathbb{O}^{\prime}}$ as follows:

$$
\begin{aligned}
\mathbb{C}[\overline{\mathbb{O}}] & \simeq\left(\mathcal{H}\left(K_{\mathbb{C}}\right) \otimes \mathbb{C}\left[\overline{\mathbb{O}^{\prime}}\right]\right)^{K_{\mathbb{C}}^{\prime}} \\
& \simeq \sum_{\tau_{1}, \tau_{2} \in R\left(K_{\mathbb{C}}^{\prime}\right)}^{\oplus} \operatorname{Hom}_{K_{\mathbb{C}}^{\prime}}\left(\tau_{1} \otimes \tau_{2}^{*}, \mathbb{C}\left[\overline{\mathbb{O}^{\prime}}\right]\right) \otimes\left(\sigma^{+}\left(\tau_{1}^{*}\right) \otimes \sigma^{-}\left(\tau_{2}\right)\right),
\end{aligned}
$$

where $\sigma^{+}\left(\tau_{1}^{*}\right) \otimes \sigma^{-}\left(\tau_{2}\right)$ is an irreducible representation of $K_{\mathbb{C}}=K_{\mathbb{C}}^{+} \times K_{\mathbb{C}}^{-}$given in (2.4), and $K_{\mathbb{C}}$ acts on the space of multiplicities $\operatorname{Hom}_{K_{\mathbb{C}}^{\prime}}\left(\tau_{1} \otimes \tau_{2}^{*}, \mathbb{C}\left[\overline{\mathbb{O}^{\prime}}\right]\right)$ trivially.

Proof. The assertion that $\overline{\mathbb{O}}$ is an affine quotient follows from general theory of affine quotient maps because $\varphi$ itself is a quotient map, and $\Xi\left(\mathbb{O}^{\prime}\right)$ is a $K_{\mathbb{C}}^{\prime}$-invariant, affine closed subvariety of $W$ (cf. [20, Prop. 3.3]).

Next we prove the statement on the module structure of $\mathbb{C}[\overline{\mathbb{O}}]$. By Theorem 2.5 . we have

$$
\begin{aligned}
\mathbb{C}\left[\Xi\left(\mathbb{O}^{\prime}\right)\right] & \simeq \mathbb{C}\left[W \times_{\mathfrak{s}^{\prime}} \overline{\mathbb{O}^{\prime}}\right]=\mathbb{C}[W] \otimes_{\mathbb{C}\left[\mathfrak{s}^{\prime}\right]} \mathbb{C}\left[\overline{\mathbb{O}^{\prime}}\right] \\
& \simeq\left(\mathbb{C}\left[W^{+}\right] \otimes \mathbb{C}\left[W^{-}\right]\right) \otimes_{\left.{\mathbb{C}\left[\mathfrak{s}_{+}^{\prime}\right.}^{\prime}\right] \otimes \mathbb{C}\left[\mathfrak{s}_{-}^{\prime}\right]} \mathbb{C}\left[\overline{\mathbb{O}^{\prime}}\right] \\
& \simeq\left(\mathbb{C}\left[W^{+}\right] \otimes_{\mathbb{C}\left[\mathfrak{s}_{+}^{\prime}\right]} \mathbb{C}\left[\overline{\mathbb{Q}^{\prime}}\right]\right) \otimes_{\mathbb{C}\left[\overline{\mathbb{Q}^{\prime}}\right]}\left(\mathbb{C}\left[W^{-}\right] \otimes_{\left.\mathbb{C}_{\left[\mathfrak{s}_{-}^{\prime}\right.}\right]} \mathbb{C}\left[\overline{\mathbb{Q}^{\prime}}\right]\right)
\end{aligned}
$$


Note that $\mathbb{C}\left[W^{ \pm}\right] \simeq \mathcal{H}\left(K_{\mathbb{C}}^{ \pm}\right) \otimes \mathbb{C}\left[W^{ \pm}\right]^{K_{\mathbb{C}}^{ \pm}}$and $\mathbb{C}\left[W^{ \pm}\right]^{K_{\mathbb{C}}^{ \pm}} \simeq \mathbb{C}\left[\mathfrak{s}_{ \pm}^{\prime}\right]$ by Theorem 2.3. Therefore we have

$$
\mathbb{C}\left[W^{+}\right] \otimes_{\mathbb{C}\left[\mathfrak{s}_{+}^{\prime}\right]} \mathbb{C}\left[\overline{\mathbb{O}^{\prime}}\right] \simeq\left(\mathcal{H}\left(K_{\mathbb{C}}^{+}\right) \otimes \mathbb{C}\left[W^{+}\right]^{K_{\mathbb{C}}^{+}}\right) \otimes_{\mathbb{C}\left[\mathfrak{s}_{+}^{\prime}\right]} \mathbb{C}\left[\overline{\mathbb{O}^{\prime}}\right] \simeq \mathcal{H}\left(K_{\mathbb{C}}^{+}\right) \otimes \mathbb{C}\left[\overline{\mathbb{O}^{\prime}}\right],
$$

and (2.6) becomes

$$
\left(\mathcal{H}\left(K_{\mathbb{C}}^{+}\right) \otimes \mathbb{C}\left[\overline{\bar{O}^{\prime}}\right]\right) \otimes_{\mathbb{C}\left[\overline{\mathbb{O}^{\prime}}\right]}\left(\mathcal{H}\left(K_{\mathbb{C}}^{-}\right) \otimes \mathbb{C}\left[\overline{\mathbb{O}^{\prime}}\right]\right) \simeq\left(\mathcal{H}\left(K_{\mathbb{C}}^{+}\right) \otimes \mathcal{H}\left(K_{\mathbb{C}}^{-}\right)\right) \otimes \mathbb{C}\left[\overline{\mathbb{O}^{\prime}}\right] .
$$

Since $\varphi$ is an affine quotient map by the action of $K_{\mathbb{C}}^{\prime}$, we have

$$
\begin{aligned}
\mathbb{C}[\overline{\mathbb{O}}] & \simeq \mathbb{C}\left[\Xi\left(\mathbb{O}^{\prime}\right)\right]^{K_{\mathbb{C}}^{\prime}} \simeq\left(\left(\mathcal{H}\left(K_{\mathbb{C}}^{+}\right) \otimes \mathcal{H}\left(K_{\mathbb{C}}^{-}\right)\right) \otimes \mathbb{C}\left[\overline{\mathbb{O}^{\prime}}\right]\right)^{K_{\mathbb{C}}^{\prime}} \\
& \simeq \sum_{\tau_{1}, \tau_{2} \in R\left(K_{\mathbb{C}}^{\prime}\right)}^{\oplus}\left(\left(\sigma^{+}\left(\tau_{1}^{*}\right) \otimes \tau_{1}^{*}\right) \otimes\left(\sigma^{-}\left(\tau_{2}\right) \otimes \tau_{2}\right) \otimes \mathbb{C}\left[\overline{\mathbb{O}^{\prime}}\right]\right)^{K_{\mathbb{C}}^{\prime}} \\
& \simeq \sum_{\tau_{1}, \tau_{2} \in R\left(K_{\mathbb{C}}^{\prime}\right)}^{\oplus}\left(\tau_{1}^{*} \otimes \tau_{2} \otimes \mathbb{C}\left[\overline{\mathbb{O}^{\prime}}\right]\right)^{K_{\mathbb{C}}^{\prime}} \otimes\left(\sigma^{+}\left(\tau_{1}^{*}\right) \otimes \sigma^{-}\left(\tau_{2}\right)\right) \\
& \simeq \sum_{\tau_{1}, \tau_{2} \in R\left(K_{\mathbb{C}}^{\prime}\right)}^{\oplus} \operatorname{Hom}_{K_{\mathbb{C}}^{\prime}}\left(\tau_{1} \otimes \tau_{2}^{*}, \mathbb{C}\left[\overline{\mathbb{O}^{\prime}}\right]\right) \otimes\left(\sigma^{+}\left(\tau_{1}^{*}\right) \otimes \sigma^{-}\left(\tau_{2}\right)\right) .
\end{aligned}
$$

The theorem follows.

Let us briefly describe our orbit correspondence in terms of signed Young diagrams. See also [24, 25], 6].

Since our $G$ or $G^{\prime}$ is either $S p(2 n, \mathbb{R}), O(p, q), U(m, n), S p(p, q)$ or $O^{*}(2 n)$, their

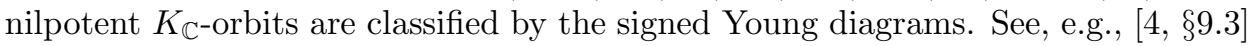
for the classification in the case of classical Lie algebras. We reproduce it for the readers' convenience.

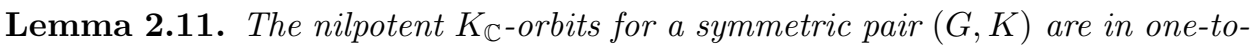
one correspondence with the following signed Young diagrams:

(1) $G=S p(2 n, \mathbb{R})$ : diagrams of size $2 n$ with any signature in which odd rows begin with + and occur with even multiplicity.

(2) $G=O(p, q)$ : diagrams of size $p+q$ with signature $(p, q)$ in which even rows begin with + and occur with even multiplicity.

(3) $G=U(m, n)$ : diagrams of size $m+n$ with signature $(m, n)$.

(4) $G=O^{*}(2 n)$ : diagrams of size $n$ with any signature in which odd rows begin with + .

(5) $G=S p(p, q)$ : diagrams of size $p+q$ with signature $(p, q)$ in which even rows begin with + .

Proposition 2.12. Let $\mathbb{O}^{\prime}$ be a nilpotent $K_{\mathbb{C}}^{\prime}$-orbit in $\mathcal{N}\left(\mathfrak{s}^{\prime}\right)$, and $\mathbb{O}=\theta\left(\mathbb{O}^{\prime}\right)$ its theta lift. If $\mathbb{O}^{\prime}$ corresponds to a signed Young diagram $\lambda^{\prime}, \mathbb{O}$ corresponds to $\lambda$ which is obtained by adding one box to the right end of each row (including empty ones) of $\lambda^{\prime}$. The signature of the added boxes are completely determined by the requirement that $\lambda$ is a signed Young diagram for $(G, K)$.

Proof. We note that the stable range condition ensures that we can always make $\lambda$ out of $\lambda^{\prime}$ by adding a box in each row. Then the proof is based on a case-bycase calculation, using explicit realization of moment maps in the Appendix. Since we know that $\varphi\left(\psi^{-1}\left(\overline{\mathbb{O}^{\prime}}\right)\right)=\overline{\mathbb{O}}$, we only need to take a representative of $\mathbb{O}^{\prime}$ and examine a generic element in $\varphi\left(\psi^{-1}\left(\overline{\mathbb{O}^{\prime}}\right)\right)$. 


\section{LiFTING OF HOLOMORPHIC NILPOTENT ORBITS}

We apply Theorem 2.10 to the lifting of the so-called holomorphic nilpotent orbits. In the process, we get a family of spherical orbits and prove normality of the closure of certain lifted orbits. This application reproduces some of our previous results $([19,[20,22])$ as well. In the following, we use the notation in Theorem 2.10 freely.

Let us consider the orbit decomposition of $\mathfrak{s}_{+}^{\prime}$ by the adjoint action of $K_{\mathbb{C}}^{\prime}$. Any $K_{\mathbb{C}^{-}}^{\prime}$ orbit in $\mathfrak{s}_{+}^{\prime}$ is clearly nilpotent. We call these nilpotent orbits holomorphic. It is well known that $\mathfrak{s}_{+}^{\prime}$ is a prehomogeneous vector space, and there exists a numbering

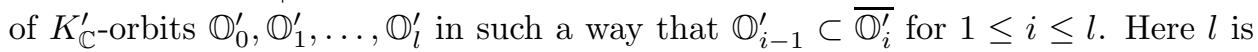
the real rank of $G^{\prime}$. As a consequence, $\mathbb{O}_{0}^{\prime}=\{0\}$ and $\overline{\mathbb{O}_{l}^{\prime}}=\mathfrak{s}_{+}^{\prime}$, i.e., $\mathbb{O}_{l}^{\prime}$ is the open dense orbit in $\mathfrak{s}_{+}^{\prime}$. The orbit $\mathbb{O}_{l}^{\prime}$ is called regular, while the orbits $\left\{\mathbb{O}_{k}^{\prime}\right\}_{0 \leq k<l}$ are called singular.

3.1. Trivial orbit. We first consider the trivial orbit $\mathbb{O}^{\prime \mathbf{1}}=\mathbb{O}_{0}^{\prime}=\{0\}$. Then, the lifted orbit $\mathbb{O}^{\mathbf{1}}=\theta\left(\mathbb{O}^{\mathbf{1}}\right)$ is a two-step nilpotent orbit in $\mathcal{N}(\mathfrak{s})$ (cf. 19). The orbit $\mathbb{0}^{1}$ corresponds to the following signed Young diagrams:

$$
\begin{array}{ll}
O(p, q): \quad \lambda=\left[(+-)^{n}(-+)^{n}(+)^{p-2 n}(-)^{q-2 n}\right] \\
U(p, q): \quad \lambda=\left[(+-)^{m}(-+)^{n}(+)^{p-(m+n)}(-)^{q-(m+n)}\right], \\
S p(p, q): \quad \lambda=\left[(+-)^{n}(+)^{p-n}(-)^{q-n}\right] .
\end{array}
$$

We have

Theorem 3.1. The group $K_{\mathbb{C}}$ acts on $\overline{\mathbb{O}^{1}}$ multiplicity-freely. As a $K_{\mathbb{C}}=K_{\mathbb{C}}^{+} \times K_{\mathbb{C}}^{-}$module, we have

$$
\mathbb{C}\left[\overline{\mathbb{O}^{1}}\right] \simeq \sum_{\tau \in R\left(K_{\mathbb{C}}^{\prime}\right)}^{\oplus} \sigma^{+}\left(\tau^{*}\right) \otimes \sigma^{-}(\tau)
$$

Proof. Since $\mathbb{C}\left[\mathbb{O}^{\prime 1}\right]=\mathbb{C}$, by Theorem 2.10 , we get

$$
\mathbb{C}\left[\overline{\mathbb{O}^{1}}\right] \simeq \sum_{\tau_{1}, \tau_{2} \in R\left(K_{\mathbb{C}}^{\prime}\right)}^{\oplus} \operatorname{Hom}_{K_{\mathbb{C}}^{\prime}}\left(\tau_{1} \otimes \tau_{2}^{*}, \mathbb{C}\right) \otimes\left(\sigma^{+}\left(\tau_{1}^{*}\right) \otimes \sigma^{-}\left(\tau_{2}\right)\right)
$$

But, by Schur's lemma, the multiplicity $\operatorname{Hom}_{K_{\mathbb{C}}^{\prime}}\left(\tau_{1} \otimes \tau_{2}^{*}, \mathbb{C}\right)$ is not zero if and only if $\tau_{1}=\tau_{2}$, in which case it is $\mathbb{C}$, i.e., the multiplicity is one.

Recall that an orbit $\mathbb{O}$ is called spherical, or more precisely $K_{\mathbb{C}}$-spherical, if a Borel subgroup of $K_{\mathbb{C}}$ has a dense orbit in $\mathbb{O}$. This is equivalent to saying that $K_{\mathbb{C}}$ acts on $\overline{\mathbb{O}}$ multiplicity-freely (i.e., $\mathbb{C}[\overline{\mathbb{O}}]$ decomposes without multiplicity as a

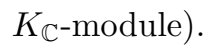

Corollary 3.2. The orbit $\mathbb{O}^{1}$ lifted from the trivial orbit is spherical. The closure $\overline{\mathbb{O}^{1}}$ is a normal variety.

Proof. The claim that $\mathbb{O}^{\mathbf{1}}$ is spherical follows directly from the above theorem. The normality follows from [29, Th. 10]. 
3.2. Regular holomorphic orbit. Let us denote by $\mathbb{O}_{l}^{\hbar}=\theta\left(\mathbb{O}_{l}^{\prime}\right)$ the theta lift from the regular holomorphic orbit $\mathbb{O}_{l}^{\prime} \subset \mathfrak{s}_{+}^{\prime}$. The orbit $\mathbb{O}_{l}^{\hbar}$ corresponds to the following signed Young diagram:

$$
\begin{array}{ll}
O(p, q): & \lambda=\left[(+-+)^{n}(+)^{p-2 n}(-)^{q-n}\right], \\
U(p, q): & \lambda=\left[(+-+)^{n}(+-)^{m-n}(+)^{p-(m+n)}(-)^{q-m}\right] \quad(m \geq n), \\
S p(p, q): & \lambda=\left\{\begin{array}{lll}
{\left[(+-+)^{(n-1) / 2}(+-)(+)^{p-n}(-)^{q-(n+1) / 2}\right]} & (n=2 l+1), \\
{\left[(+-+)^{n / 2}(+)^{p-n}(-)^{q-n / 2}\right]} & (n=2 l) .
\end{array}\right.
\end{array}
$$

For the regular orbit, we have

$$
\Xi\left(\mathbb{O}_{l}^{\prime}\right)=\psi^{-1}\left(\overline{\mathbb{O}_{l}^{\prime}}\right)=\psi^{-1}\left(\mathfrak{s}_{+}^{\prime}\right)=W^{+} \times \mathfrak{N}^{-},
$$

where $\psi^{-}=\left.\psi\right|_{W^{-}}$and $\mathfrak{N}^{-}=\left(\psi^{-}\right)^{-1}(0) \subset W^{-}$is a null cone. Thus Theorem 2.10 yields the following

Lemma 3.3. Let $\mathbb{O}_{l}^{\hbar} \subset \mathcal{N}(\mathfrak{s})$ be the theta lift of the regular holomorphic orbit $\mathbb{O}_{l}^{\prime}$, which is open dense in $\mathfrak{s}_{+}^{\prime}$. Then its closure is an affine quotient of $W^{+} \times \mathfrak{N}^{-}$by $K_{\mathbb{C}}^{\prime}$,

$$
\overline{\mathbb{O}_{l}^{\hbar}} \simeq\left(W^{+} \times \mathfrak{N}^{-}\right) / / K_{\mathbb{C}}^{\prime}
$$

Let us recall (\$1) the compact group $L^{+}$whose action on $W^{+}$commutes with that of $K^{\prime}$. In fact, $\left(L^{+}, K^{\prime}\right)$ forms a dual pair in $S p\left(W_{\mathbb{R}}^{+}\right)$. Let

$$
\mathbb{C}\left[W^{+}\right] \simeq \sum_{\tau \in \operatorname{Irr}\left(K_{\mathrm{C}}^{\prime} ; \mathbb{C}\left[W^{+}\right]\right)}^{\oplus} \rho^{+}(\tau) \otimes \tau
$$

be the decomposition of $\mathbb{C}\left[W^{+}\right]$as an $L^{+} \times K^{\prime}$-module. Here $\rho^{+}(\tau) \in \operatorname{Irr}\left(L^{+}\right)$ corresponds to $\tau \in \operatorname{Irr}\left(K^{\prime}\right)$ via the above multiplicity-free decomposition. Note that $\mathbb{C}\left[W^{+}\right] \simeq \mathcal{H}\left(K_{\mathbb{C}}^{+}\right) \otimes \mathbb{C}\left[W^{+}\right]^{K_{\mathbb{C}}^{+}}$, and so $\operatorname{Irr}\left(K_{\mathbb{C}}^{\prime} ; \mathbb{C}\left[W^{+}\right]\right)=\operatorname{Irr}\left(K_{\mathbb{C}}^{\prime} ; \mathcal{H}\left(K_{\mathbb{C}}^{+}\right)\right)=$ $R\left(K_{\mathbb{C}}^{\prime}\right)^{*}$. We may therefore rewrite (3.2) as

$$
\mathbb{C}\left[W^{+}\right] \simeq \sum_{\tau \in R\left(K_{\mathbb{C}}^{\prime}\right)}^{\oplus} \rho^{+}\left(\tau^{*}\right) \otimes \tau^{*}
$$

Note that $L^{+}$is a unitary group containing $K^{+}$in each of the three cases, hence its complexification $L_{\mathbb{C}}^{+}$is isomorphic to a general linear group. Since the action of $L_{\mathbb{C}}^{+}$commutes with that of $K_{\mathbb{C}}^{\prime}, L_{\mathbb{C}}^{+} \times K_{\mathbb{C}}^{-}$naturally acts on the affine quotient space

$$
\left(W^{+} \times \mathfrak{N}^{-}\right) / / K_{\mathbb{C}}^{\prime} \simeq \overline{\mathbb{O}_{l}^{h}}
$$

extending the action of $K_{\mathbb{C}}=K_{\mathbb{C}}^{+} \times K_{\mathbb{C}}^{-}$. Note that the orbit $\mathbb{O}_{l}^{\hbar}$ itself does not admit an action of $L_{\mathbb{C}}^{+} \times K_{\mathbb{C}}^{-}$, but its closure does.

Theorem 3.4. The group $L_{\mathbb{C}}^{+} \times K_{\mathbb{C}}^{-}$acts on the closure $\overline{\mathbb{O}_{l}^{\hbar}}$ multiplicity-freely. As a $L_{\mathbb{C}}^{+} \times K_{\mathbb{C}}^{-}$-module, we have

$$
\mathbb{C}\left[\overline{\mathbb{O}_{l}^{\hbar}}\right] \simeq \sum_{\tau \in R\left(K_{\mathrm{C}}^{\prime}\right)}^{\oplus} \rho^{+}\left(\tau^{*}\right) \otimes \sigma^{-}(\tau) .
$$

Consequently, the closure $\overline{\mathbb{O}_{l}^{\hbar}}$ is $L_{\mathbb{C}}^{+} \times K_{\mathbb{C}}^{-}$-spherical, and it is a normal variety. 
Proof. We have

$$
\begin{aligned}
\mathbb{C}\left[\overline{\mathbb{O}_{l}^{\hbar}}\right] & \simeq\left(\mathbb{C}\left[W^{+}\right] \otimes \mathbb{C}\left[\mathfrak{N}^{-}\right]\right)^{K_{\mathbb{C}}^{\prime}} \\
& \simeq\left(\left(\sum_{\tau_{1} \in R\left(K_{\mathbb{C}}^{\prime}\right)}^{\oplus} \rho^{+}\left(\tau_{1}^{*}\right) \otimes \tau_{1}^{*}\right) \otimes\left(\sum_{\tau_{2} \in R\left(K_{\mathbb{C}}^{\prime}\right)}^{\oplus} \sigma^{-}\left(\tau_{2}\right) \otimes \tau_{2}\right)\right)^{K_{\mathbb{C}}^{\prime}} \\
& \simeq \sum_{\tau \in R\left(K_{\mathbb{C}}^{\prime}\right)}^{\oplus} \rho^{+}\left(\tau^{*}\right) \otimes \sigma^{-}(\tau) .
\end{aligned}
$$

The normality follows from [29, Th. 10], since $\mathbb{Z} R\left(K_{\mathbb{C}}^{\prime}\right) \cap \mathbb{Q}_{+} R\left(K_{\mathbb{C}}^{\prime}\right)=R\left(K_{\mathbb{C}}^{\prime}\right)$.

3.3. Degree of nilpotent orbits lifted from holomorphic orbits. As an application of the explicit decomposition formula of the function ring of nilpotent orbits, we give an integral formula of their degrees.

Since a nilpotent orbit $\mathbb{O} \subset \mathfrak{s}$ is a cone, we naturally consider it in a projective space $\mathbb{P}(\mathfrak{s})$. As a projective variety, the closure $\mathbb{P}(\overline{\mathbb{O}})$ in $\mathbb{P}(\mathfrak{s})$ has a degree. We shall denote this degree by $\operatorname{deg} \overline{\mathbb{O}}$ by a slight abuse of notation, and we call it the degree of the nilpotent orbit $\mathbb{O}$. We refer to [28, $\S \mathrm{V} .4 .2]$ for the definition of the degree of projective varieties. See also [8].

To state our result, let us introduce some notation. We denote by $\Delta=\Delta\left(X_{r}\right)$ an irreducible root system of type $X_{r}=A_{r-1}, B_{r}, C_{r}, D_{r}$. We realize $\Delta$ in a standard way (see [2, Planche I-IX]), and choose a positive system $\Delta^{+}$as $\Delta^{+}\left(A_{r-1}\right)=$ $\left\{\varepsilon_{i}-\varepsilon_{j} \mid 1 \leq i<j \leq r\right\} ; \Delta^{+}\left(D_{r}\right)=\left\{\varepsilon_{i} \pm \varepsilon_{j} \mid 1 \leq i<j \leq r\right\} ; \Delta^{+}\left(B_{r}\right)=$ $\Delta^{+}\left(D_{r}\right) \cup\left\{\varepsilon_{i} \mid 1 \leq i \leq r\right\} ; \Delta^{+}\left(C_{r}\right)=\Delta^{+}\left(D_{r}\right) \cup\left\{2 \varepsilon_{i} \mid 1 \leq i \leq r\right\}$. As usual, $\rho=\rho_{X_{r}}=\sum_{\alpha \in \Delta^{+}} \alpha / 2$ denotes the half sum of positive roots, and we fix an invariant inner product $\langle\alpha, \beta\rangle$ on the root space which makes $\left\{\varepsilon_{i}\right\}$ an orthonormal system. For fixed $n \leq r$, we put

$$
\begin{aligned}
& \Delta_{n}^{+}\left(X_{r}\right)=\left\{\alpha \in \Delta^{+}\left(X_{r}\right) \mid\left\langle\alpha, \varepsilon_{i}\right\rangle=0(1 \leq i \leq n)\right\}, \text { and } \\
& \Phi_{n}^{+}\left(X_{r}\right)=\Delta^{+}\left(X_{r}\right) \backslash \Delta_{n}^{+}\left(X_{r}\right),
\end{aligned}
$$

and for $m+n \leq r$,

$$
\Phi_{m, n}^{+}\left(A_{r-1}\right)=\left\{\varepsilon_{i}-\varepsilon_{j} \in \Delta^{+}\left(A_{r-1}\right) \mid i \leq m \text { or } r-n<j\right\} .
$$

We introduce the difference product $D_{n}(x)$ on a multi-variable $x=\left(x_{1}, \ldots, x_{n}\right)$ defined by

$$
D_{n}(x)=\prod_{1 \leq i<j \leq n}\left(x_{i}-x_{j}\right) .
$$

We abbreviate $D_{n}\left(x^{2}\right)=D_{n}\left(x_{1}^{2}, \ldots, x_{n}^{2}\right)$. Finally, set

$$
\Omega_{n}=\left\{x=\left(x_{i}\right)_{1 \leq i \leq n} \mid x_{i} \geq 0, \sum_{i=1}^{n} x_{i} \leq 1\right\} .
$$

Proposition 3.5. (1) Case $O(p, q) \times S p(2 n, \mathbb{R})(2 n<p, q)$ : Put $r=[p / 2], s=[q / 2]$ and $X=B$ or $D$ (respectively $Y=B$ or $D$ ) according to whether $p$ (respectively 
q) is odd or even. Then the degree of the nilpotent orbit is given by

$$
\begin{aligned}
& \operatorname{deg} \overline{\mathbb{O}^{1}}=\frac{1}{n !} \prod_{\alpha \in \Phi_{n}^{+}\left(X_{r}\right)}\left\langle\rho_{X_{r}}, \alpha\right\rangle^{-1} \prod_{\beta \in \Phi_{n}^{+}\left(Y_{s}\right)}\left\langle\rho_{Y_{s}}, \beta\right\rangle^{-1} \int_{\Omega_{n}}\left|D_{n}\left(x^{2}\right)\right|^{2} \prod_{i=1}^{n} x_{i}^{p+q-2 n} d x, \\
& \operatorname{deg} \overline{\mathbb{O}_{l}^{\hbar}}=\frac{1}{n !} \prod_{\alpha \in \Phi_{n}^{+}\left(X_{r}\right)}\left\langle\rho_{X_{r}}, \alpha\right\rangle^{-1} \prod_{\beta \in \Phi_{n}^{+}\left(A_{q-1}\right)}\left\langle\rho_{A_{q-1}}, \beta\right\rangle^{-1} \\
& \times \int_{\Omega_{n}}\left|D_{n}\left(x^{2}\right) D_{n}(x)\right| \prod_{i=1}^{n} x_{i}^{p+q-3 n} d x . \\
& \operatorname{deg} \overline{\mathbb{O}^{1}}=\frac{1}{m ! n !} \prod_{\alpha \in \Phi_{m, n}^{+}\left(A_{p-1}\right)}\left\langle\rho_{A_{p-1}}, \alpha\right\rangle^{-1} \prod_{\beta \in \Phi_{m, n}^{+}\left(A_{q-1}\right)}\left\langle\rho_{A_{q-1}}, \beta\right\rangle^{-1} \\
& \times \int_{\Omega_{m} \times \Omega_{n}}\left|D_{m}(x)\right|^{2}\left|D_{n}(y)\right|^{2} \prod_{\substack{1 \leq i \leq m \\
1 \leq j \leq n}}\left(x_{i}+y_{j}\right)^{2} \prod_{i=1}^{m} x_{i}^{p+q-2(m+n)} \prod_{j=1}^{n} y_{j}^{p+q-2(m+n)} d x d y, \\
& \operatorname{deg} \overline{\mathbb{O}_{l}^{h}}=\frac{1}{m ! n !} \prod_{\alpha \in \Phi_{m, n}^{+}\left(A_{p-1}\right)}\left\langle\rho_{A_{p-1}}, \alpha\right\rangle^{-1} \prod_{\beta \in \Phi_{m}^{+}\left(A_{q-1}\right)}\left\langle\rho_{A_{q-1}}, \beta\right\rangle^{-1} \prod_{\gamma \in \Phi_{n}^{+}\left(A_{q-1}\right)}\left\langle\rho_{A_{q-1}}, \gamma\right\rangle^{-1} \\
& \times \int_{\Omega_{m} \times \Omega_{n}}\left|D_{m}(x)\right|^{2}\left|D_{n}(y)\right|^{2} \prod_{\substack{1 \leq i \leq m \\
1 \leq j \leq n}}\left(x_{i}+y_{j}\right) \prod_{i=1}^{m} x_{i}^{p+q-(2 m+n)} \prod_{j=1}^{n} y_{j}^{p+q-(m+2 n)} d x d y .
\end{aligned}
$$

(3) Case $S p(p, q) \times O^{*}(2 n)(n \leq p, q)$ :

$$
\begin{aligned}
& \operatorname{deg} \overline{\mathbb{O}^{1}}=\frac{2^{2 n}}{n !} \prod_{\alpha \in \Phi_{n}^{+}\left(C_{p}\right)}\left\langle\rho_{C_{p}}, \alpha\right\rangle^{-1} \prod_{\beta \in \Phi_{n}^{+}\left(C_{q}\right)}\left\langle\rho_{C_{q}}, \beta\right\rangle^{-1} \\
& \times \int_{\Omega_{n}}\left|D_{n}\left(x^{2}\right)\right|^{2} \prod_{i=1}^{n} x_{i}^{2(p+q)-4 n+2} d x, \\
& \operatorname{deg} \overline{\mathbb{O}_{l}^{\hbar}}=\frac{2^{n}}{n !} \prod_{\alpha \in \Phi_{n}^{+}\left(C_{p}\right)}\left\langle\rho_{C_{p}}, \alpha\right\rangle^{-1} \prod_{\beta \in \Phi_{n}^{+}\left(A_{2 q-1}\right)}\left\langle\rho_{A_{2 q-1}}, \beta\right\rangle^{-1} \\
& \times \int_{\Omega_{n}}\left|D_{n}\left(x^{2}\right) D_{n}(x)\right| \prod_{i=1}^{n} x_{i}^{2(p+q)-3 n+1} d x .
\end{aligned}
$$

Proof. Since we know the $K_{\mathbb{C}}$-decomposition of the regular function ring, we can formally express the Poincaré series by using Weyl's dimension formula. The degree $e$ is encoded in the top term of the Hilbert polynomial $H_{\overline{\mathbb{O}}}(t)$, whose degree coincides with $d=\operatorname{dim} \mathbb{P}(\overline{\mathbb{O}})$. Thus, we have $e=d ! \lim _{t \rightarrow \infty} H_{\overline{\mathbb{O}}}(t) / t^{d}$, which is essentially expressed by a Riemann integral (the limit of a Riemann sum). This machinery is explained in [15, §2.2]. We omit the details.

Let us evaluate the following integral, which appears in the above proposition.

Theorem 3.6. Let $\kappa$ be a complex number with a positive real part. Then

$$
\frac{1}{n !} \int_{\Omega_{n}} D_{n}\left(x^{2}\right) D_{n}(x) \prod_{i=1}^{n} x_{i}^{\kappa-1} d x=\frac{2^{n(n-1) / 2} \prod_{i=0}^{n-1} i ! \Gamma(\kappa+2 i)}{\Gamma(3 n(n-1) / 2+n \kappa+1)} .
$$

Proof. Setting $f(x)=D_{n}\left(x^{2}\right) D_{n}(x) \prod_{i=1}^{n} x_{i}^{\kappa-1}$ and using its homogeneity, we see that

$$
\Gamma(a+1) \int_{\Omega_{n}} f(x) d x=\int_{\Omega_{n} \times(0, \infty)} f(x) s^{a} e^{-s} d x d s
$$


where $a=3 n(n-1) / 2+n \kappa$. By making a change of variables: $y_{i}=s x_{i}, t=$ $\left(1-\sum_{i=1}^{n} x_{i}\right) s$, we obtain

$$
\text { (3.6) }=\int_{(0, \infty)^{n+1}} f(y) e^{-\sum_{i=1}^{n} y_{i}} e^{-t} d y d t=\int_{(0, \infty)^{n}} f(y) e^{-\sum_{i=1}^{n} y_{i}} d y .
$$

By expanding the term $D_{n}\left(y^{2}\right) D_{n}(y)$ in the expression of $f(y)$, (3.6) becomes

$$
\begin{aligned}
& \sum_{\sigma, \tau} \operatorname{sgn}(\sigma) \operatorname{sgn}(\tau) \prod_{i=1}^{n} \int_{0}^{\infty} y_{i}^{2(\sigma(i)-1)+(\tau(i)-1)+\kappa-1} e^{-y_{i}} d y_{i} \\
& =n ! \operatorname{det}(\Gamma(2 i+j+\kappa-3))_{i, j=1, \ldots, n} \\
& =n ! \prod_{i=0}^{n-1} \Gamma(\kappa+2 i) \times \operatorname{det}\left((2 i+\kappa-2)_{j-1}\right)_{i, j=1, \ldots, n},
\end{aligned}
$$

where $(c)_{k}=c(c+1) \cdots(c+k-1)$. The last determinant is computed to be $2^{n(n-1) / 2} \prod_{i=1}^{n-1} i$ !. Formula (3.5) thus follows.

Remark 3.7. By a similar argument, we have

$$
\begin{aligned}
\frac{1}{n !} \int_{\Omega_{n}} D_{n}\left(x^{2}\right)^{2} \prod_{i=1}^{n} x_{i}^{\kappa-1} d x= & \frac{\prod_{i=0}^{n-1} \Gamma(\kappa+2 i)}{\Gamma(2 n(n-1)+n \kappa+1)} \\
& \times \operatorname{det}\left((\kappa+2 i-2)_{2 j-2}\right)_{i, j=1, \ldots, n} .
\end{aligned}
$$

The last determinant depends on $\kappa$ polynomially and seems to have no simple expression. This suggests that $\overline{\mathbb{O}^{1}}$ may have a more complicated structure than that of $\overline{\mathbb{O}_{l}^{\hbar}}$.

\section{Appendix}

In this Appendix, we will give an explicit construction of moment maps $\varphi$ and $\psi$, and establish some basic properties of null cones and harmonics.

4.1. $O(p, q) \times S p(2 n, \mathbb{R})(2 n<\min (p, q))$. This case is already treated in [19]. For convenience of the readers, we reproduce the synopsis of the arguments, and at the same time we improve some of the statements there.

Put $G=O(p, q)$ and $G^{\prime}=S p(2 n, \mathbb{R})$, and assume the stable range condition $2 n<p, q$. We denote

$$
\begin{aligned}
& M=U(p, q) \quad \supset \quad G=O(p, q) \\
& L=L^{+} \times L^{-} \quad \supset \quad K=K^{+} \times K^{-} \\
& =U(p) \times U(q) \quad=O(p) \times O(q)
\end{aligned}
$$

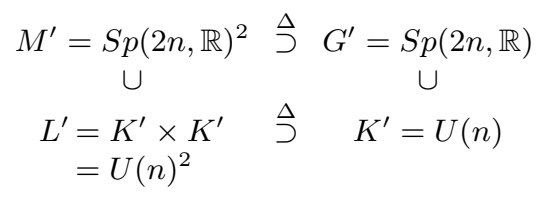

In this diagram, the vertical containment means respectively maximal compact subgroups, and $\Delta$ denotes the diagonal embedding. Put

$$
\begin{aligned}
W & =M_{p+q, n}(\mathbb{C})=\left\{\left(\begin{array}{l}
A \\
B
\end{array}\right) \mid A \in M_{p, n}(\mathbb{C}), B \in M_{q, n}(\mathbb{C})\right\} \\
& =W^{+} \oplus W^{-}=M_{p, n}(\mathbb{C}) \oplus M_{q, n}(\mathbb{C}) .
\end{aligned}
$$


The complexification $K_{\mathbb{C}}=O(p, \mathbb{C}) \times O(q, \mathbb{C})$ acts on $W$ via the left multiplication, and $K_{\mathbb{C}}^{\prime}=G L_{n}(\mathbb{C})$ acts on $W$ as

$$
g \cdot\left(\begin{array}{l}
A \\
B
\end{array}\right)=\left(\begin{array}{c}
A^{t} g \\
B g^{-1}
\end{array}\right), \quad g \in G L_{n}(\mathbb{C}) .
$$

We fix a Cartan decomposition $\mathfrak{g}=\mathfrak{k} \oplus \mathfrak{s}\left(\right.$ resp. $\left.\mathfrak{g}^{\prime}=\mathfrak{k}^{\prime} \oplus \mathfrak{s}^{\prime}\right)$ as

$$
\begin{aligned}
& \mathfrak{g}=\mathfrak{o}(p+q, \mathbb{C})=\left(\begin{array}{c|c}
\operatorname{Alt}_{p}(\mathbb{C}) & 0 \\
\hline 0 & \operatorname{Alt}_{q}(\mathbb{C})
\end{array}\right) \oplus\left(\begin{array}{c|c}
0 & M_{p, q}(\mathbb{C}) \\
\hline{ }^{t} M_{p, q}(\mathbb{C}) & 0
\end{array}\right)=\mathfrak{k} \oplus \mathfrak{s}, \\
& \mathfrak{g}^{\prime}=\mathfrak{s p}(2 n, \mathbb{C})=\left(\begin{array}{c|c|c}
M_{n}(\mathbb{C}) & 0 \\
\hline 0 & -{ }^{t} M_{n}(\mathbb{C})
\end{array}\right) \oplus\left(\begin{array}{c|c}
0 & \operatorname{Sym}_{n}(\mathbb{C}) \\
\hline \operatorname{Sym}_{n}(\mathbb{C}) & 0
\end{array}\right)=\mathfrak{k}^{\prime} \oplus \mathfrak{s}^{\prime} .
\end{aligned}
$$

Thus, we can identify $\mathfrak{s}=M_{p, q}(\mathbb{C})$ and $\mathfrak{s}^{\prime}=\mathfrak{s}_{+}^{\prime} \oplus \mathfrak{s}_{-}^{\prime}=\operatorname{Sym}_{n}(\mathbb{C}) \oplus \operatorname{Sym}_{n}(\mathbb{C})$. The moment maps are explicitly given by

$$
\varphi: W \ni\left(\begin{array}{l}
A \\
B
\end{array}\right) \longmapsto A^{t} B \in \mathfrak{s}, \quad \psi: W \ni\left(\begin{array}{l}
A \\
B
\end{array}\right) \longmapsto\left({ }^{t} A A,{ }^{t} B B\right) \in \mathfrak{s}^{\prime} .
$$

These maps are clearly $K_{\mathbb{C}} \times K_{\mathbb{C}}^{\prime}$-equivariant if we define the trivial $K_{\mathbb{C}^{-} \text {-action (resp. }}$

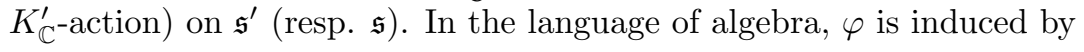

$$
\varphi^{*}\left(z_{i, j}\right)=\sum_{k=1}^{n} a_{i, k} b_{j, k}, \quad Z=\left(z_{i, j}\right) \in \mathfrak{s},\left(\begin{array}{c}
A \\
B
\end{array}\right)=\left(\begin{array}{c}
\left(a_{i, j}\right) \\
\left(b_{i, j}\right)
\end{array}\right) \in W,
$$

and it is well known that $\varphi^{*}\left(z_{i, j}\right)$ 's generate $G L_{n}$-invariants $\mathbb{C}[W]^{K_{\mathrm{C}}^{\prime}}$. This shows that $\varphi: W \rightarrow \mathfrak{s}$ is an affine quotient map onto its closed image: $\mathfrak{s} \supset \varphi(W) \simeq$ $W / / K_{\mathbb{C}}^{\prime}$. Similarly, $\psi$ is induced by

$$
\begin{gathered}
\psi^{*}\left(x_{i, j}\right)=\sum_{k=1}^{p} a_{k, i} a_{k, j}, \quad X=\left(x_{i, j}\right) \in \mathfrak{s}_{+}^{\prime} ; \\
\psi^{*}\left(y_{i, j}\right)=\sum_{l=1}^{q} b_{l, i} b_{l, j}, Y=\left(y_{i, j}\right) \in \mathfrak{s}_{-}^{\prime} .
\end{gathered}
$$

Again, $\psi\left(x_{i, j}\right)^{*}$ 's and $\psi\left(y_{i, j}\right)^{*}$ 's generate the $O(p, \mathbb{C}) \times O(q, \mathbb{C})$-invariants $\mathbb{C}[W]^{K_{\mathbb{C}}}$, which proves that $\psi: W \rightarrow \mathfrak{s}^{\prime}$ is an affine quotient map. By the assumption of the stable range, $2 n<p, q$, it is easy to see that $\psi: W \rightarrow \mathfrak{s}^{\prime}$ is a surjection, hence $\mathfrak{s}^{\prime} \simeq W / / K_{\mathbb{C}}$.

Let us consider the null cones

$$
\mathfrak{N}=\psi^{-1}(0) \subset W, \quad \mathfrak{N}^{ \pm}=\left(\psi^{ \pm}\right)^{-1}(0) \subset W^{ \pm},
$$

where $\psi^{ \pm}: W^{ \pm} \rightarrow \mathfrak{s}_{ \pm}^{\prime}$ is the restriction of $\psi$ to $W^{ \pm}$.

Proposition 4.1. Assume the stable range condition $2 n<p, q$.

(1) The null cone $\mathfrak{N}=\mathfrak{N}^{+} \times \mathfrak{N}^{-}$is an irreducible normal variety, which is of complete intersection in the set theoretic sense.

(2) There are finitely many $K_{\mathbb{C}} \times K_{\mathbb{C}^{-}}^{\prime}$ orbits in $\mathfrak{N}$, which are completely classified by the ranks of each component in $\mathfrak{N}^{ \pm}$. Among them, there is an open dense $K_{\mathbb{C}} \times K_{\mathbb{C}}^{\prime}$-orbit $\mathcal{O}_{n, n}$, which is a single $K_{\mathbb{C} \text {-orbit. Moreover, the singular locus }}$ coincides with $\mathfrak{N} \backslash \mathcal{O}_{n, n}$, which is of codimension $\geq 2$. 
(3) The regular function ring $\mathbb{C}[\mathfrak{N}]$ is isomorphic to the harmonics $\mathcal{H}\left(K_{\mathbb{C}}\right) \simeq$ $\mathcal{H}^{+}\left(K_{\mathbb{C}}^{+}\right) \otimes \mathcal{H}^{-}\left(K_{\mathbb{C}}^{-}\right)$as a $K_{\mathbb{C}} \times K_{\mathbb{C}}^{\prime}$-module. Its decomposition is explicitly given as $\mathbb{C}\left[\mathfrak{N}^{+}\right] \simeq \mathcal{H}^{+}\left(K_{\mathbb{C}}^{+}\right) \simeq \sum_{\lambda \in \mathcal{P}_{n}}^{\oplus} \sigma_{\lambda}^{(p) *} \otimes \tau_{\lambda}^{(n) *}, \quad \mathbb{C}\left[\mathfrak{N}^{-}\right] \simeq \mathcal{H}^{-}\left(K_{\mathbb{C}}^{-}\right) \simeq \sum_{\lambda \in \mathcal{P}_{n}}^{\oplus} \sigma_{\lambda}^{(q)} \otimes \tau_{\lambda}^{(n)}$,

where $\mathcal{P}_{n}$ denotes the set of all the partitions of length $\leq n$, and $\tau_{\lambda}^{(n)}\left(\right.$ resp. $\left.\sigma_{\lambda}^{(p)}\right)$ is an irreducible finite dimensional representation of $G L_{n}($ resp. $O(p, \mathbb{C}))$ of highest weight $\lambda$.

Remark 4.2. (1) In [19, the orbit decomposition of the null cone is carried out for $K_{\mathbb{C}} \times L_{\mathbb{C}}^{\prime}$, though it is more natural to consider $K_{\mathbb{C}} \times K_{\mathbb{C}}^{\prime}$-orbits. In fact, their orbits are the same (under the assumption of stable range). Also, in [19, the normality is proved when $2 n+2 \leq p, q$. Here we improve it, and there is no more restriction other than the stable range condition.

(2) It is rather subtle to specify the representation $\sigma_{\lambda}^{(p)}$ because $O(p, \mathbb{C})$ is not connected. For this, see [12, §3.6.2].

Proof. It is straightforward to check the claim for the $K_{\mathbb{C}} \times K_{\mathbb{C}}^{\prime}$-orbit decomposition in (4.1) directly by calculation. Let us denote $\mathcal{O}_{r, s}=\mathcal{O}_{r}^{+} \times \mathcal{O}_{s}^{-} \in \mathfrak{N}$, where

$$
\begin{aligned}
& \mathcal{O}_{r}^{+}=\left\{A \in M_{p, n} \mid{ }^{t} A A=0, \operatorname{rank} A=r\right\} \subset \mathfrak{N}^{+}, \\
& \mathcal{O}_{s}^{-}=\left\{B \in M_{q, n} \mid{ }^{t} B B=0, \operatorname{rank} B=s\right\} \subset \mathfrak{N}^{-} .
\end{aligned}
$$

Then $\mathcal{O}_{r}^{+}$is a $K_{\mathbb{C}}^{+} \times K_{\mathbb{C}}^{\prime}$-orbit, and $\mathcal{O}_{r, s}$ is a single $K_{\mathbb{C}} \times K_{\mathbb{C}^{-}}^{\prime}$-rbit. Since they are classified by rank, the orbit of the largest possible rank $\mathcal{O}_{n, n}$ is open dense in $\mathfrak{N}$. This implies that $\mathfrak{N}=\overline{\mathcal{O}_{n, n}}$ is irreducible. In fact, $\mathcal{O}_{n, n}$ is a single $K_{\mathbb{C}^{-} \text {orbit. This }}$ follows from the Witt theorem.

Explicit calculation of the rank of differentials of the defining equations of $\psi^{*}\left(x_{i j}\right)$ 's and $\psi^{*}\left(y_{i j}\right)$ 's tells us that a point from $\mathfrak{N} \backslash \mathcal{O}_{n, n}$ is singular. Since

$$
\operatorname{dim} \mathfrak{N}^{+}=n p-\frac{n(n+1)}{2}, \quad \operatorname{dim} \mathfrak{N}^{-}=n q-\frac{n(n+1)}{2},
$$

we have codim $\mathfrak{N}=n(n+1)$, which is equal to the number of the defining equations. This proves that $\mathfrak{N}$ is of complete intersection.

The dimension of the $K_{\mathbb{C}}^{+} \times K_{\mathbb{C}^{-}}^{\prime}$-rbit in $\mathfrak{N}^{+}$of rank $r$ is given by

$$
\operatorname{dim} \mathcal{O}_{r}^{+}=r(p+n)-r^{2}-\frac{r(r+1)}{2}
$$

From this formula, we can easily show that $\operatorname{codim}\left(\mathfrak{N} \backslash \mathcal{O}_{n, n}\right) \geq 2$.

By Kostant theorem (see [3, Th. 2.2.11], for example), we know that the defining radical ideal of $\mathfrak{N}$ is generated by $\psi^{*}\left(x_{i j}\right)$ 's and $\psi^{*}\left(y_{i j}\right)$ 's, which are basic invariants of $O(p, \mathbb{C}) \times O(q, \mathbb{C})$. This means that

$$
\mathbb{C}[\mathfrak{N}]=\mathbb{C}[W] /\left(\psi^{*}\left(x_{i j}\right), \psi^{*}\left(y_{i j}\right)\right)=\mathbb{C}[W] / \mathbb{C}[W] \cdot \mathbb{C}[W]_{+}^{K_{\mathbb{C}}} \simeq \mathcal{H}\left(K_{\mathbb{C}}\right) .
$$

The decomposition of $\mathcal{H}\left(K_{\mathbb{C}}\right)$ is given in [12, Th. 3.7.3.1 \& Cor. 3.7.3.6]. See also [7] and [14. By the same theorem of Kostant, the null cone $\mathfrak{N}$ is normal. Also, $\mathfrak{N}^{ \pm}$ are normal varieties. 
4.2. $U(p, q) \times U(m, n)(m+n \leq \min (p, q))$. Put $G=U(p, q)$ and $G^{\prime}=U(m, n)$, and assume the stable range condition $m+n \leq p, q$. We denote

$$
\begin{array}{ccc}
M=U(p, q)^{2} & \supset & G=U(p, q) \\
\cup & & \cup \\
L=L^{+} \times L^{-}=U(p)^{2} \times U(q)^{2} & \doteq & K=K^{+} \times K^{-}=U(p) \times U(q)
\end{array}
$$

Here $\Delta$ denotes the diagonal embedding. Similarly, we denote $M^{\prime}, G^{\prime}, L^{\prime}, K^{\prime}$ replacing $p$ and $q$ by $m$ and $n$ respectively. Put

$$
\begin{aligned}
W & =M_{p+q, m+n}(\mathbb{C})=\left\{\left(\begin{array}{ll}
A & B \\
C & D
\end{array}\right) \mid A \in M_{p, m}, B \in M_{p, n}, C \in M_{q, m}, D \in M_{q, n}\right\} \\
& =W^{+} \oplus W^{-}=M_{p, m+n}(\mathbb{C}) \oplus M_{q, m+n}(\mathbb{C}) .
\end{aligned}
$$

Then $K_{\mathbb{C}}=G L_{p}(\mathbb{C}) \times G L_{q}(\mathbb{C})$ and $K_{\mathbb{C}}^{\prime}=G L_{m}(\mathbb{C}) \times G L_{n}(\mathbb{C})$ act on $W$ as

$$
\begin{aligned}
& \left(\left(g_{1}, g_{2}\right),\left(h_{1}, h_{2}\right)\right) \cdot\left(\begin{array}{ll}
A & B \\
C & D
\end{array}\right)=\left(\begin{array}{cc}
g_{1} A^{t} h_{1} & { }^{t} g_{1}{ }^{-1} B h_{2}^{-1} \\
{ }^{t} g_{2}{ }^{-1} C h_{1}^{-1} & g_{2} D^{t} h_{2}
\end{array}\right), \\
& \left(g_{1}, g_{2}\right) \in G L_{p}(\mathbb{C}) \times G L_{q}(\mathbb{C}), \quad\left(h_{1}, h_{2}\right) \in G L_{m}(\mathbb{C}) \times G L_{n}(\mathbb{C}) .
\end{aligned}
$$

We fix a Cartan decomposition $\mathfrak{g}=\mathfrak{k} \oplus \mathfrak{s}$ as

$$
\mathfrak{g}=\mathfrak{g l}_{p+q}(\mathbb{C})=\left(\begin{array}{c|c}
M_{p}(\mathbb{C}) & 0 \\
\hline 0 & M_{q}(\mathbb{C})
\end{array}\right) \oplus\left(\begin{array}{c|c}
0 & M_{p, q}(\mathbb{C}) \\
\hline M_{q, p}(\mathbb{C}) & 0
\end{array}\right)=\mathfrak{k} \oplus \mathfrak{s} .
$$

The Cartan decomposition $\mathfrak{g}^{\prime}=\mathfrak{k}^{\prime} \oplus \mathfrak{s}^{\prime}$ is chosen similarly. We identify

$$
\mathfrak{s}=M_{p, q}(\mathbb{C}) \oplus M_{q, p}(\mathbb{C}), \quad \mathfrak{s}^{\prime}=M_{m, n}(\mathbb{C}) \oplus M_{n, m}(\mathbb{C}) .
$$

The moment maps $\varphi$ and $\psi$ are explicitly given by

$$
\begin{gathered}
\varphi: W \ni\left(\begin{array}{ll}
A & B \\
C & D
\end{array}\right) \longmapsto\left(A^{t} C, D^{t} B\right) \in \mathfrak{s}, \\
\psi: W \ni\left(\begin{array}{ll}
A & B \\
C & D
\end{array}\right) \longmapsto\left({ }^{t} A B,{ }^{t} D C\right) \in \mathfrak{s}^{\prime} .
\end{gathered}
$$

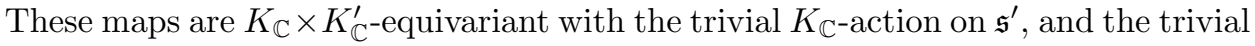
$K_{\mathbb{C}}^{\prime}$-action on $\mathfrak{s}$ respectively. The maps $\varphi$ and $\psi$ are almost the same. Therefore we will treat only the map $\psi$ in the following.

The map $\psi$ induces an algebra homomorphism $\psi: \mathbb{C}\left[\mathfrak{s}^{\prime}\right] \rightarrow \mathbb{C}[W]$ by

$$
\psi^{*}\left(x_{i, j}\right)=\sum_{k=1}^{p} a_{k, i} b_{k, j} ; \quad \psi^{*}\left(y_{i, j}\right)=\sum_{l=1}^{q} c_{l, j} d_{l, i},
$$

where $\left(x_{i, j}\right)$ and $\left(y_{i, j}\right)$ are coordinate functions on $(X, Y) \in M_{m, n}(\mathbb{C}) \times M_{n, m}(\mathbb{C})=$ $\mathfrak{s}^{\prime}$. Then the $G L_{p^{-}}$-invariants (resp. $G L_{q}$-invariants) on $\mathbb{C}\left[W^{+}\right]$(resp. $\mathbb{C}\left[W^{-}\right]$) are generated by $\psi\left(x_{i, j}\right)^{*}$ 's (resp. $\psi\left(y_{i, j}\right)^{*}$ 's). Hence $\psi: W \rightarrow \mathfrak{s}^{\prime}$ is an affine quotient map by $K_{\mathbb{C}}$, which is surjective under the condition of the stable range. Note that $\varphi$ is not surjective in general.

Let us consider the null cones

$$
\mathfrak{N}=\psi^{-1}(0) \subset W, \quad \mathfrak{N}^{ \pm}=\left(\psi^{ \pm}\right)^{-1}(0) \subset W^{ \pm},
$$

where $\psi^{ \pm}: W^{ \pm} \rightarrow \mathfrak{s}_{ \pm}^{\prime}$ is the restriction of $\psi$ to $W^{ \pm}$. 
Proposition 4.3. Assume the stable range condition $m+n \leq \min (p, q)$.

(1) The null cone $\mathfrak{N}=\mathfrak{N}^{+} \times \mathfrak{N}^{-}$is an irreducible normal variety, which is of complete intersection in the set theoretic sense.

(2) There are finitely many $K_{\mathbb{C}} \times K_{\mathbb{C}}^{\prime}$-orbits in $\mathfrak{N}$, which are completely classified by the ranks of each component in $\mathfrak{N}^{ \pm}$. Among them, there is an open dense $K_{\mathbb{C}} \times K_{\mathbb{C}}^{\prime}$-orbit $\mathcal{O}_{m, n}$, which is a single $K_{\mathbb{C} \text {-orbit. } \text { Moreover, the singular locus }}$ is of codimension $\geq 2$.

(3) The regular function ring $\mathbb{C}[\mathfrak{N}]$ is isomorphic to the harmonics $\mathcal{H}\left(K_{\mathbb{C}}\right) \simeq$ $\mathcal{H}^{+}\left(K_{\mathbb{C}}^{+}\right) \otimes \mathcal{H}^{-}\left(K_{\mathbb{C}}^{-}\right)$as a $K_{\mathbb{C}} \times K_{\mathbb{C}}^{\prime}$-module. Its decomposition is explicitly given as

$$
\begin{aligned}
& \mathbb{C}\left[\mathfrak{N}^{+}\right] \simeq \mathcal{H}^{+}\left(K_{\mathbb{C}}^{+}\right) \simeq \sum_{\alpha \in \mathcal{P}_{m}, \beta \in \mathcal{P}_{n}}^{\oplus} \tau_{\alpha \odot \beta}^{(p)}{ }^{*} \otimes\left(\tau_{\alpha}^{(m) *} \otimes \tau_{\beta}^{(n)}\right), \\
& \mathbb{C}\left[\mathfrak{N}^{-}\right] \simeq \mathcal{H}^{-}\left(K_{\mathbb{C}}^{-}\right) \simeq \sum_{\gamma \in \mathcal{P}_{m}, \delta \in \mathcal{P}_{n}}^{\oplus} \tau_{\gamma \odot \delta}^{(q)} \otimes\left(\tau_{\gamma}^{(m)} \otimes \tau_{\delta}^{(n) *}\right),
\end{aligned}
$$

where, for partitions $\alpha \in \mathcal{P}_{m}$ and $\beta \in \mathcal{P}_{n}$, we denote

$$
\alpha \odot \beta=\alpha \odot_{p} \beta=\left(\alpha_{1}, \alpha_{2}, \ldots, \alpha_{m}, 0, \ldots, 0,-\beta_{n}, \ldots,-\beta_{1}\right) \in \mathbb{Z}^{p} ;
$$

and $\tau_{\lambda}^{(p)}$ is an irreducible finite dimensional representation of $G L_{p}$ of highest weight $\lambda$.

Proof. Put $\mathcal{O}_{r, s}^{+}=\left\{(A, B) \in M_{p, m+n} \mid{ }^{t} A B=0, \operatorname{rank} A=r, \operatorname{rank} B=s\right\} \subset \mathfrak{N}^{+}$. Then $\mathcal{O}_{r, s}^{+}$is a $K_{\mathbb{C}}^{+} \times K_{\mathbb{C}}^{\prime}$-orbit, and $\operatorname{dim} \mathcal{O}_{r, s}^{+}=r(m+p)+s(n+p)+r s-(r+s)^{2}$. It is easy to see that $\mathcal{O}_{m, n}^{+} \subset \mathfrak{N}^{+}$is an open dense orbit and $\operatorname{dim} \mathfrak{N}^{+}=\operatorname{dim} \mathcal{O}_{m, n}^{+}=$ $p(m+n)-m n$, which means $\mathfrak{N}^{+}$is of complete intersection. The singular locus is given by

$$
\overline{\mathcal{O}_{m-1, n-1}^{+}}=\coprod_{r \leq m-1, s \leq n-1} \mathcal{O}_{r, s}^{+} \subset \mathfrak{N}^{+},
$$

which is of codimension $\geq 2$. The rest of the proof is similar to that of Proposition 4.1.

The decomposition of harmonics is given in [12, Th. 2.5.4].

4.3. $S p(p, q) \times O^{*}(2 n)(n \leq \min (p, q))$. Put $G=S p(p, q)$ and $G^{\prime}=O^{*}(2 n)$, and assume the stable range condition $n \leq p, q$. We denote

$$
\begin{aligned}
& M=\underset{\cup}{U(2 p, 2 q)} \supset \quad G=\underset{\cup}{S p(p, q)} \quad M^{\prime}=\underset{\cup}{O^{*}(2 n)^{2}} \stackrel{\searrow}{\supset} G^{\prime}=O^{*}(2 n)
\end{aligned}
$$

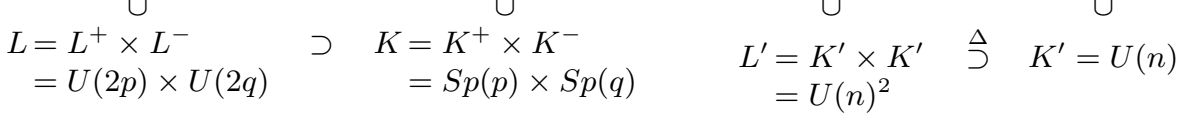

Put

$$
\begin{aligned}
W & =M_{2 p+2 q, n}(\mathbb{C})=\left\{\left(\begin{array}{l}
A \\
B
\end{array}\right) \mid A \in M_{2 p, n}(\mathbb{C}), B \in M_{2 q, n}(\mathbb{C})\right\} \\
& =W^{+} \oplus W^{-}=M_{2 p, n}(\mathbb{C}) \oplus M_{2 q, n}(\mathbb{C}) .
\end{aligned}
$$

The complexified groups $K_{\mathbb{C}}=S p(2 p, \mathbb{C}) \times S p(2 q, \mathbb{C})$ and $K_{\mathbb{C}}^{\prime}=G L_{n}(\mathbb{C})$ act on $W$ as

$$
\left(\begin{array}{l}
A \\
B
\end{array}\right) \mapsto\left(\begin{array}{c}
k_{1} A^{t} g \\
{ }^{t} k_{2}{ }^{-1} B g^{-1}
\end{array}\right), \quad\left(k_{1}, k_{2}\right) \in S p(2 p, \mathbb{C}) \times S p(2 q, \mathbb{C}),
$$


We fix Cartan decompositions $\mathfrak{g}=\mathfrak{k} \oplus \mathfrak{s}$ and $\mathfrak{g}^{\prime}=\mathfrak{k}^{\prime} \oplus \mathfrak{s}^{\prime}$ as

$$
\begin{aligned}
\mathfrak{g} & =\mathfrak{s p}(2 p+2 q, \mathbb{C})=\left(\begin{array}{c|c}
\mathfrak{s p}(2 p, \mathbb{C}) & 0 \\
\hline 0 & \mathfrak{s p}(2 q, \mathbb{C})
\end{array}\right) \oplus\left(\begin{array}{c|c}
0 & M_{2 p, 2 q} \\
\hline J_{q}{ }^{t} M_{2 p, 2 q} J_{p} & 0
\end{array}\right) \\
\quad=\mathfrak{k} \oplus \mathfrak{s}, \quad J_{p}=\left(\begin{array}{cc}
0 & 1_{p} \\
-1_{p} & 0
\end{array}\right), & \mathfrak{g}^{\prime}=\mathfrak{o}(2 n, \mathbb{C})=\left(\begin{array}{c|c}
M_{n}(\mathbb{C}) & 0 \\
\hline 0 & -{ }^{t} M_{n}(\mathbb{C})
\end{array}\right) \oplus\left(\begin{array}{c|c}
0 & \operatorname{Alt}_{n}(\mathbb{C}) \\
\hline \operatorname{Alt}_{n}(\mathbb{C}) & 0
\end{array}\right)=\mathfrak{k}^{\prime} \oplus \mathfrak{s}^{\prime} .
\end{aligned}
$$

Thus, we can identify $\mathfrak{s}=M_{2 p, 2 q}(\mathbb{C})$ and $\mathfrak{s}^{\prime}=\mathfrak{s}_{+}^{\prime} \oplus \mathfrak{s}_{-}^{\prime}=\operatorname{Alt}_{n}(\mathbb{C}) \oplus \operatorname{Alt}_{n}(\mathbb{C})$. The moment maps are explicitly given by

$$
\varphi: W \ni\left(\begin{array}{l}
A \\
B
\end{array}\right) \longmapsto A^{t} B \in \mathfrak{s}, \quad \psi: W \ni\left(\begin{array}{l}
A \\
B
\end{array}\right) \longmapsto\left({ }^{t} A J_{p} A,{ }^{t} B J_{q} B\right) \in \mathfrak{s}^{\prime} .
$$

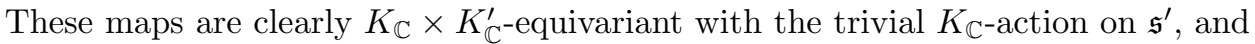
the trivial $K_{\mathbb{C}^{-}}^{\prime}$ action on $\mathfrak{s}$ respectively. They induce algebra morphisms $\varphi^{*}$ and $\psi^{*}$ :

$$
\begin{aligned}
\varphi^{*}\left(z_{i, j}\right) & =\sum_{k=1}^{n} a_{i, k} b_{j, k}, \quad Z=\left(z_{i, j}\right) \in M_{2 p, 2 q} \\
A & =\left(a_{i, j}\right) \in M_{2 p, n}, B=\left(b_{i, j}\right) \in M_{2 q, n} \\
\psi^{*}\left(x_{i, j}\right) & =-\sum_{k=1}^{p} a_{k+p, i} a_{k, j}+\sum_{k=1}^{p} a_{k, i} a_{k+p, j}, \quad X=\left(x_{i, j}\right) \in \operatorname{Alt}_{n} \\
\psi^{*}\left(y_{i, j}\right) & =-\sum_{l=1}^{q} b_{l+q, i} b_{l, j}+\sum_{l=1}^{q} b_{l, i} b_{l+q, j}, \quad Y=\left(y_{i, j}\right) \in \operatorname{Alt}_{n} .
\end{aligned}
$$

Classical invariant theory tells us that $\varphi^{*}\left(z_{i, j}\right)$ 's generate the $G L_{n}$-invariants $\mathbb{C}[W]^{K_{\mathbb{C}}^{\prime}}$, and that $\psi\left(x_{i, j}\right)^{*}$ 's (resp. $\psi\left(y_{i, j}\right)^{*}$ 's) generate the $K_{\mathbb{C}}^{+}=S p(2 p, \mathbb{C}$ )invariants $\mathbb{C}\left[W^{+}\right]^{K_{\mathbb{C}}^{+}}$(resp. $K_{\mathbb{C}}^{-}=S p(2 q, \mathbb{C})$-invariants $\mathbb{C}\left[W^{-}\right]^{K_{\mathbb{C}}^{-}}$). This shows that $\varphi$ and $\psi$ are affine quotient maps. By the assumption of the stable range, $n \leq p, q$, the map $\psi: W \rightarrow \mathfrak{s}^{\prime}$ is a surjection, and we have $\mathbb{C}\left[\mathfrak{s}^{\prime}\right] \simeq \mathbb{C}[W]^{K_{\mathbb{C}}}, \mathbb{C}\left[\mathfrak{s}_{ \pm}^{\prime}\right] \simeq$ $\mathbb{C}\left[W^{ \pm}\right]^{K_{\mathbb{C}}^{ \pm}}$.

Let us define the null cones by

$$
\mathfrak{N}=\psi^{-1}(0) \subset W, \quad \mathfrak{N}^{ \pm}=\left(\psi^{ \pm}\right)^{-1}(0) \subset W^{ \pm},
$$

where $\psi^{ \pm}: W^{ \pm} \rightarrow \mathfrak{s}_{ \pm}^{\prime}$ is the restriction of $\psi$ to $W^{ \pm}$.

Proposition 4.4. Assume the stable range condition $n \leq p, q$.

(1) The null cone $\mathfrak{N}=\mathfrak{N}^{+} \times \mathfrak{N}^{-}$is an irreducible normal variety, which is of complete intersection in the set theoretical sense.

(2) There are finitely many $K_{\mathbb{C}} \times K_{\mathbb{C}}^{\prime}$-orbits in $\mathfrak{N}$, which are completely classified by the ranks of each component in $\mathfrak{N}^{ \pm}$. The full rank orbit $\mathcal{O}_{n, n}$ is an open dense

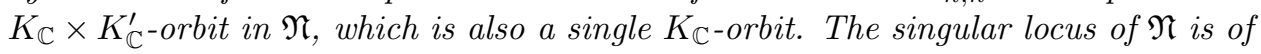
codimension $\geq 2$. 
(3) The regular function ring $\mathbb{C}[\mathfrak{N}]$ is isomorphic to the harmonics $\mathcal{H}\left(K_{\mathbb{C}}\right) \simeq$ $\mathcal{H}^{+}\left(K_{\mathbb{C}}^{+}\right) \otimes \mathcal{H}^{-}\left(K_{\mathbb{C}}^{-}\right)$as a $K_{\mathbb{C}} \times K_{\mathbb{C}}^{\prime}$-module, and we have

$\mathbb{C}\left[\mathfrak{N}^{+}\right] \simeq \mathcal{H}^{+}\left(K_{\mathbb{C}}^{+}\right) \simeq \sum_{\lambda \in \mathcal{P}_{n}}^{\oplus} \sigma_{\lambda}^{(p) *} \otimes \tau_{\lambda}^{(n) *} ; \quad \mathbb{C}\left[\mathfrak{N}^{-}\right] \simeq \mathcal{H}^{-}\left(K_{\mathbb{C}}^{-}\right) \simeq \sum_{\lambda \in \mathcal{P}_{n}}^{\oplus} \sigma_{\lambda}^{(q)} \otimes \tau_{\lambda}^{(n)}$,

where $\sigma_{\lambda}^{(p)}$ denotes an irreducible finite dimensional representation of $\operatorname{Sp}(2 p, \mathbb{C})$ with highest weight $\lambda \in \mathcal{P}_{n}$.

Proof. Put

$$
\begin{aligned}
& \mathcal{O}_{r}^{+}=\left\{A \in M_{2 p, n} \mid{ }^{t} A J_{p} A=0, \operatorname{rank} A=r\right\} \subset \mathfrak{N}^{+}, \\
& \mathcal{O}_{s}^{-}=\left\{B \in M_{2 q, n} \mid{ }^{t} B J_{q} B=0, \operatorname{rank} B=s\right\} \subset \mathfrak{N}^{-} .
\end{aligned}
$$

Then $\mathcal{O}_{r, s}=\mathcal{O}_{r}^{+} \times \mathcal{O}_{s}^{-}$is a single $K_{\mathbb{C}} \times K_{\mathbb{C}^{-}}^{\prime}$ orbit and

$$
\operatorname{dim} \mathcal{O}_{r}^{+}=r(2 p+n)-r^{2}-\frac{r(r-1)}{2}, \quad \operatorname{dim} \mathcal{O}_{s}^{+}=s(2 q+n)-s^{2}-\frac{s(s-1)}{2} .
$$

It is easy to show that $\mathcal{O}_{n}^{ \pm} \subset \mathfrak{N}^{ \pm}$is an open dense orbit and

$$
\operatorname{dim} \mathfrak{N}^{+}=\operatorname{dim} \mathcal{O}_{n}^{+}=2 p n-\frac{n(n-1)}{2}, \quad \operatorname{dim} \mathfrak{N}^{-}=\operatorname{dim} \mathcal{O}_{n}^{-}=2 q n-\frac{n(n-1)}{2},
$$

which tells us that $\mathfrak{N}$ (resp. $\mathfrak{N}^{ \pm}$) is of complete intersection. The singular locus is given by

$$
\coprod_{r \leq n-2} \mathcal{O}_{r}^{ \pm} \subset \mathfrak{N}^{ \pm}
$$

which is of codimension $\geq 2$. The rest of the proof is similar to that of Proposition 4.1 .

The structure of harmonics is given in [12, Th. 3.8.6.2].

\section{REFERENCES}

[1] Walter Borho and Hanspeter Kraft, Über Bahnen und deren Deformationen bei linearen Aktionen reduktiver Gruppen. Comment. Math. Helv. 54 (1979), no. 1, 61-104. MR522032 (82m:14027)

[2] N. Bourbaki, Groupes et algéres de Lie. Chapitre IV - VI. Hermann, Paris 1968. MR.0240238 $(39: 1590)$

[3] N. Chriss and V. Ginzburg, Representation theory and complex geometry, Birkhäuser Boston, Boston, MA, 1997, ISBN: 0-8176-3792-3. MR1433132 (98i:22021)

[4] D. Collingwood and W. McGovern, Nilpotent orbits in semisimple Lie algebras, Reinhold, Van Nostrand, New York 1993. MR1251060 (94j:17001)

[5] A. Daszkiewicz, W. Kraśkiewicz and T. Przebinda, Nilpotent orbits and complex dual pairs, Jour. Algebra 190, (1997), 518-539. MR1441961 (98b:20063)

[6] A. Daszkiewicz, W. Kraśkiewicz and T. Przebinda, Dual pairs and Kostant-Sekiguchi correspondence. II. Classification of nilpotent elements, preprint.

[7] Stephen S. Gelbart, A theory of Stiefel harmonics. Trans. Amer. Math. Soc. 192 (1974), 29-50. MR0425519(54:13474)

[8] Robin Hartshorne, Algebraic geometry. Graduate Texts in Mathematics, No. 52. SpringerVerlag, New York-Heidelberg, 1977. MR0463157 (57:3116)

[9] R. Howe, $\theta$-series and invariant theory, Proc. Symp. Pure Math. 33, Ameri. Math. Soc., Providence, 1979. MR0546602 (81f:22034)

[10] R. Howe, Remarks on classical invariant theory. Trans. Amer. Math. Soc. 313 (1989), no. 2, 539-570. Erratum to: "Remarks on classical invariant theory". Trans. Amer. Math. Soc. 318 (1990), no. 2, 823. MR0986027 (90h:22015a)

[11] R. Howe, Transcending classical invariant theory. J. Amer. Math. Soc. 2 (1989), no. 3, 535552. MR0985172 (90k:22016) 
[12] R. Howe, Perspectives on invariant theory: Schur duality, multiplicity-free actions and beyond, in The Schur lectures (1992) (Tel Aviv), 1-182, Bar-Ilan Univ., Ramat Gan, 1995. MR.1321638 (96e:13006)

[13] J.-S. Huang and J.-S. Li, Unipotent representations attached to spherical nilpotent orbits, Amer. Jour. Math. 121 (1999), no. 3, 497-517. MR1738410(2000m:22018)

[14] M. Kashiwara and M. Vergne, On the Segal-Shale-Weil representations and harmonic polynomials, Invent. Math. 44 (1978), no. 1, 1-47. MR0463359 (57:3311)

[15] Shohei Kato and Hiroyuki Ochiai, The degrees of orbits of the multiplicity free actions. Astérisque No. 273, (2001), 139-158. MR.1845716

[16] Donald R. King, Classification of spherical nilpotent orbits in complex symmetric space, J. Lie Theory 14 (2004), 339-370. MR2066860

[17] B. Kostant, Lie group representations on polynomial rings, Amer. Jour. Math. 85 (1963), 327-404. MR158024(28:1252)

[18] J.-S. Li, Singular unitary representations of classical groups, Invent. Math., 97 (1989), 237255. MR001840 (90h:22021)

[19] Kyo Nishiyama, Multiplicity-free actions and the geometry of nilpotent orbits. Math. Ann., 318 (2000), 777-793. MR.1802510 (2002e:22016)

[20] Kyo Nishiyama, Theta lifting of two-step nilpotent orbits for the pair $O(p, q) \times S p(2 n, \mathbb{R})$. In "Infinite Dimensional Harmonic Analysis", Transaction of a Japanese-German Symposium (Kyoto, September 1999), Kyoto 1999, pp. 278-289. MR.1851930(2002g:22026)

[21] Kyo Nishiyama, Hiroyuki Ochiai, and Kenji Taniguchi, Bernstein degree and associated cycles of Harish-Chandra modules-Hermitian symmetric case. Astérisque No. 273, (2001), 13-80. MR1845714

[22] Kyo Nishiyama and C.-B. Zhu, Theta lifting of holomorphic discrete series: the case of $U(p, q) \times U(n, n)$. Trans. AMS. 353 (2001), 3327-3345. MR.1828608 (2002e:22017)

[23] Kyo Nishiyama and C.-B. Zhu, Theta lifting of unitary lowest weight modules and their associated cycles, Duke Math. J. 125 (2004), 415-465. MR2166751

[24] Takuya Ohta, Nilpotent orbits of $Z_{4}$-graded Lie algebra and geometry of the moment maps associated to the dual pair $(U(p, q), U(r, s))$, preprint.

[25] Takuya Ohta, Nilpotent orbits of $Z_{4}$-graded Lie algebra and geometry of the moment maps associated to the dual pairs $(O(p, q), S p(2 n, \mathbb{R}))$ and $\left(O^{*}(2 n), S p(p, q)\right)$, in preparation.

[26] V. L. Popov and E. B. Vinberg, Invariant theory, in "Algebraic geometry. IV.", Encyclopaedia of Mathematical Sciences, 55. Springer-Verlag, Berlin, 1994, pp. 123-284.

[27] T. Przebinda, Characters, dual pairs, and unitary representations, Duke Math. J. 69 (1993), no. 3, 547-594. MR1208811 (94i:22036)

[28] Igor R. Shafarevich, Basic algebraic geometry. 1. Varieties in projective space. Second edition. Springer-Verlag, Berlin, 1994. MR.1328833 (95m:14001)

[29] E. B. Vinberg and V. L. Popov, A certain class of quasihomogeneous affine varieties. Izv. Akad. Nauk SSSR Ser. Mat. 36 (1972), 749-764. MR0313260 (47:1815)

[30] D. A. Vogan, Jr., Associated varieties and unipotent representations, in Harmonic analysis on reductive groups (Brunswick, ME, 1989), 315-388, Progr. Math. 101, Birkhäuser, Boston, Boston, MA, 1991. MR 1168491 (93k:22012)

[31] H. Weyl, The classical groups, Princeton University Press, 1946.

Department of Mathematics, Graduate School of Science, Kyoto University, Sakyo, КҮОТО 606-8502, JAPAN

E-mail address: kyo@math.kyoto-u.ac.jp

Department of Mathematics, Nagoya University, Nagoya, 464-8602, Japan

E-mail address: ochiai@math.nagoya-u.ac.jp

Department of Mathematics, National University of Singapore, 2 Science Drive 2, SiNGAPORE 117543

E-mail address: matzhucb@nus.edu.sg 